\title{
The $\beta$ Pictoris association low-mass members: Membership assessment, rotation period distribution, and dependence on multiplicity ${ }^{\star}$
}

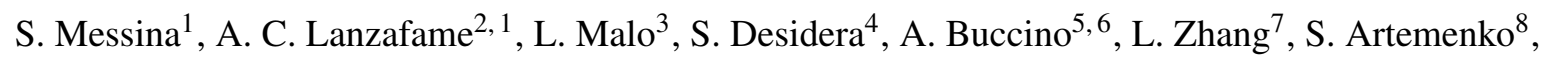
M. Millward ${ }^{9}$, and F.-J. Hambsch ${ }^{10,11}$

1 INAF-Catania Astrophysical Observatory, via S. Sofia 78, 95123 Catania, Italy

e-mail: sergio.messina@oact.inaf.it

2 Universitá di Catania, Dipartimento di Fisica e Astronomia, Sezione Astrofisica, via S. Sofia 78, 95123 Catania, Italy

3 Canada-France-Hawaii Telescope, 65-1238 Mamalahoa Hwy, Kamuela, HI 96743, USA

4 INAF-Osservatorio Astronomico di Padova, Vicolo dell'Osservatorio 5, 35122 Padova, Italy

5 Instituto de Astronomía y Física del Espacio (IAFE-CONICET), 1053 Buenos Aires, Argentina

6 Departamento de Física, FCEN-Universidad de Buenos Aires, 1053 Buenos Aires, Argentina

7 Department of Physics, College of Science, Guizhou University, 550025 Guiyang, PR China

8 Research Institute Crimean Astrophysical Observatory, 298409 Nauchny, Crimea

9 York Creek Observatory, Georgetown, Tasmania, Australia

${ }_{10}^{10}$ Remote Observatory Atacama Desert (ROAD), Vereniging Voor Sterrenkunde (VVS), Oude Bleken 12, 2400 Mol, Belgium

11 American Association of Variable Star Observers (AAVSO), Cambridge, MA 02138, USA

Received 16 January 2017 / Accepted 5 July 2017

\begin{abstract}
Context. Low-mass members of young loose stellar associations and open clusters exhibit a wide spread of rotation periods. Such a spread originates from the distributions of masses and initial rotation periods. However, multiplicity can also play a significant role. Aims. We aim to investigate the role played by physical companions in multiple systems in shortening the primordial disk lifetime, anticipating the rotation spin up with respect to single stars.

Methods. We have compiled the most extensive list to date of low-mass bona fide and candidate members of the young 25-Myr $\beta$ Pictoris association. We have measured from our own photometric time series or from archival time series the rotation periods of almost all members. In a few cases the rotation periods were retrieved from the literature. We used updated $U V W X Y Z$ components to assess the membership of the whole stellar sample. Thanks to the known basic properties of most members we built the rotation period distribution distinguishing between bona fide members and candidate members and according to their multiplicity status.

Results. We find that single stars and components of multiple systems in wide orbits ( $>80 \mathrm{AU})$ have rotation periods that exhibit a well defined sequence arising from mass distribution with some level of spread likely arising from initial rotation period distribution. All components of multiple systems in close orbits $(<80 \mathrm{AU})$ have rotation periods that are significantly shorter than their equal-mass single counterparts. For these close components of multiple systems a linear dependence of rotation rate on separation is only barely detected. A comparison with the younger $13 \mathrm{Myr} h$ Per cluster and with the older 40-Myr open clusters and stellar associations NGC 2547, IC 2391, Argus, and IC 2602 and the 130-Myr Pleiades shows that whereas the evolution of F-G stars is well reproduced by angular momentum evolution models, this is not the case for the slow $\mathrm{K}$ and early-M stars. Finally, we find that the amplitude of their light curves is correlated neither with rotation nor with mass.

Conclusions. Once single stars and wide components of multiple systems are separated from close components of multiple systems, the rotation period distributions exhibit a well defined dependence on mass that allows us to make a meaningful comparison with similar distributions of either younger or older associations and clusters. Such cleaned distributions allow us to use the stellar rotation period meaningfully as an age indicator for $\mathrm{F}$ and $\mathrm{G}$ type stars.
\end{abstract}

Key words. stars: activity - stars: late-type - starspots - binaries: close - open clusters and associations: individual: $\beta$ Pictoris stars: rotation

\section{Introduction}

$\beta$ Pictoris is a nearby young loose stellar association. Its members have an average distance from the Sun of about $40 \pm 17 \mathrm{pc}$ and an age of about 25 \pm 3 Myr (Messina et al. 2016a, hereafter Paper I). Youth and proximity make this association a special benchmark in stellar astrophysics studies. In fact, the young

\footnotetext{
* Tables 2 and 3 are only available at the CDS via anonymous ftp to cdsarc.u-strasbg. fr (130.79.128.5) or via http://cdsarc.u-strasbg.fr/viz-bin/qcat?J/A+A/607/A3
}

age secures the presence of interesting circumstellar environments in many members, where disks and planetary systems can be discovered. The proximity allows us to spatially resolve them giving effective possibility to study disk morphology and the planetary system's architecture. The A3V star $\beta$ Pictoris, from which the association takes the name, is one such example (see, e.g., Chauvin et al. 2012). Youth, vicinity, and brightness of its members explain why this association has been included in many studies searching for very low-mass stellar and planetary companions, as well as studies aiming to accurately 
measure element abundances, magnetic activity, and kinematics. Among the many studies available, here we mention those aimed at searching for planetary companions and disks like the SEEDS project (Strategic Exploration of Exoplanets and Disks with Subaru; Brandt et al. 2014), The Gemini/NICI planet-finding campaign (Biller et al. 2013), SPHERE (Spectro-Polarimetric Highcontrast Exoplanet REsearch, Beuzit et al. 2008), and $\mathrm{NaCo}$ Large Program (Desidera et al. 2015); those aimed at searching for and characterizing new members, such as the SACY project (Search for Associations Containing Young stars; Torres et al. 2006, 2008; da Silva et al. 2009; Elliott et al. 2014), The solar neighborhood investigation (Riedel et al. 2014), the BANYAN project (Bayesian Analysis for Nearby Young Associations; Malo et al. 2013, 2014a,b), and many other membership investigations (see, e.g., Lépine \& Simon 2009; Kiss et al. 2011; Schlieder et al. 2010, 2012; Shkolnik et al. 2012; Malo et al. 2013, 2014a,b) resulting in a significantly increased number, by about a factor three, of confirmed and new candidate members, with respect to the association members detected in discovery studies (e.g., Zuckerman et al. 2001).

The first comprehensive rotational investigation of the lowmass (spectral types $\mathrm{F}$ to $\mathrm{M}$ ) members of the $\beta$ Pictoris association was carried out by Messina et al. (2010, 2011). They measured the rotation periods of 33 on a list of 38 among confirmed and candidate members compiled from Zuckerman \& Song (2004) and Torres et al. (2006, 2008).

The rotational properties of the $\beta$ Pictoris association represent a key information for a number of studies concerning, for example, the pre-main-sequence (PMS) angular momentum evolution of low-mass stars (see Spada et al. 2011; Gallet \& Bouvier 2013, 2015), the effect of rotation on lithium depletion at young ages (Pallavicini et al. 1993; Bouvier et al. 2016; Messina et al. 2016a), the impact of photoevaporation and binary encounters on the primordial disk life time (Olczak et al. 2010; Throop \& Bally 2008) and the timescale of the star-disk locking phase, which can all be probed by means of the star's rotation (see, e.g., Messina et al. 2014, 2015a,b), as well as the implication for the formation of planets around binaries (see, e.g., Alexander 2012).

Considering the importance of the $\beta$ Pictoris association for these studies, the increased number of newly discovered members, and the fact that the basic properties of many members have been better established over time (thanks to their brightness), we realized that the time was ripe for carrying out a new rotational study on this enlarged sample. The results of this extensive study were presented in the catalog of photometric rotational periods by Messina et al. (2017, hereafter Paper II) containing the photometric rotational periods of 112 low-mass members and candidate members of the $\beta$ Pictoris association. These rotation periods were used to explore the rotation-lithium connection and to obtain an improved age estimate of the $\beta$ Pictoris association using the lithium depletion boundary method (Paper I). In the present study (Paper III), we aim at exploiting this catalog of rotation periods to investigate the distribution of rotation periods versus mass and the role played by multiplicity, which is known for most members, in determining the wide spread of rotation periods observed in this and other young loose associations.

In Sect. 2, we present the up-to-date and most complete sample of members. In Sect.3, we discuss on the basic properties, color and rotation period, that are used in our analysis. In Sect. 4, we present the results of our period search. In Sect. 5 , we assess the membership of the whole sample using updated space and velocity components. In Sect. 6, we discuss the rotation period distribution and present new results on the impact of multiplicity on the rotation evolution. In Sect. 7, we make a comparison of the rotation period distribution with those of younger and of older open clusters and associations. Dependence of photospheric activity, as measured from light curve amplitude, on rotation and mass is discussed in Sect. 8. In Sect. 9, we give our conclusions.

\section{Sample description}

The present study is based on the catalog of photometric rotational periods of low-mass members and candidate members of the $\beta$ Pictoris association presented in Paper II. Briefly, we have carried out an extensive search in the literature to retrieve all members of the $\beta$ Pictoris association. We compiled a list of 117 among bona fide and candidate members, with spectral types later than about F3V, from the following major studies: Torres et al. (2006, 2008), Lépine \& Simon (2009), Kiss et al. (2011), Schlieder et al. (2010, 2012), Shkolnik et al. (2012), Malo et al. (2013, 2014a,b), and other studies detailed for each member in Paper II. Stars of earlier spectral types were excluded from our sample since the photometric rotation period to be measured requires the presence of a detectable level of magnetic activity (more specifically, of light rotational modulation by surface temperature inhomogeneities with amplitude of several millimag at least). This circumstance generally occurs in late spectral type stars that have an external convection zone, which allows for the production of magnetic fields and is subjected to magnetic braking.

We measured the rotation periods of 112 out of 117 stars either from our own photometric monitoring or from photometric time series in public archives, or we retrieved these periods from the literature.

Information on individual stars either from our own analysis or from the literature and references can be retrieved in $\mathrm{Pa}-$ per II. Information on the membership is not homogeneous for all the targets either for the number of studies or for the methods. For example, we found more than four membership studies for 52 targets, whereas only one membership study for 15 targets. For this reason, in Sect. 4, we present the results of our membership study based on updated space and velocity components and lithium equivalent width (EW).

The single or binary nature of our targets is based on the available RV measurements and direct imaging studies, which are referenced for each target in Appendix A of Paper II. We note that not all stars with constant RV have been observed with high-contrast direct imaging. Therefore, for these stars, despite the RV constancy, we cannot rule out the presence of a wide orbit companion. However, even if this is the case, their rotational properties are indistinguishable from those of known wide binaries (see Sect. 5). Targets with not determined either single or binary nature from RV studies are flagged with a symbol "?" in the last column of Table A.1. The complete target list is reported in Table A.1.

\section{Target properties}

\subsection{Colors}

Our aim is to investigate the distribution of the rotation periods versus stellar mass and the impact of multiplicity on the observed rotation period spread. The stellar mass for the majority of our target stars has to be derived from a comparison with evolutionary mass tracks at the age of the $\beta$ Pictoris association. The 

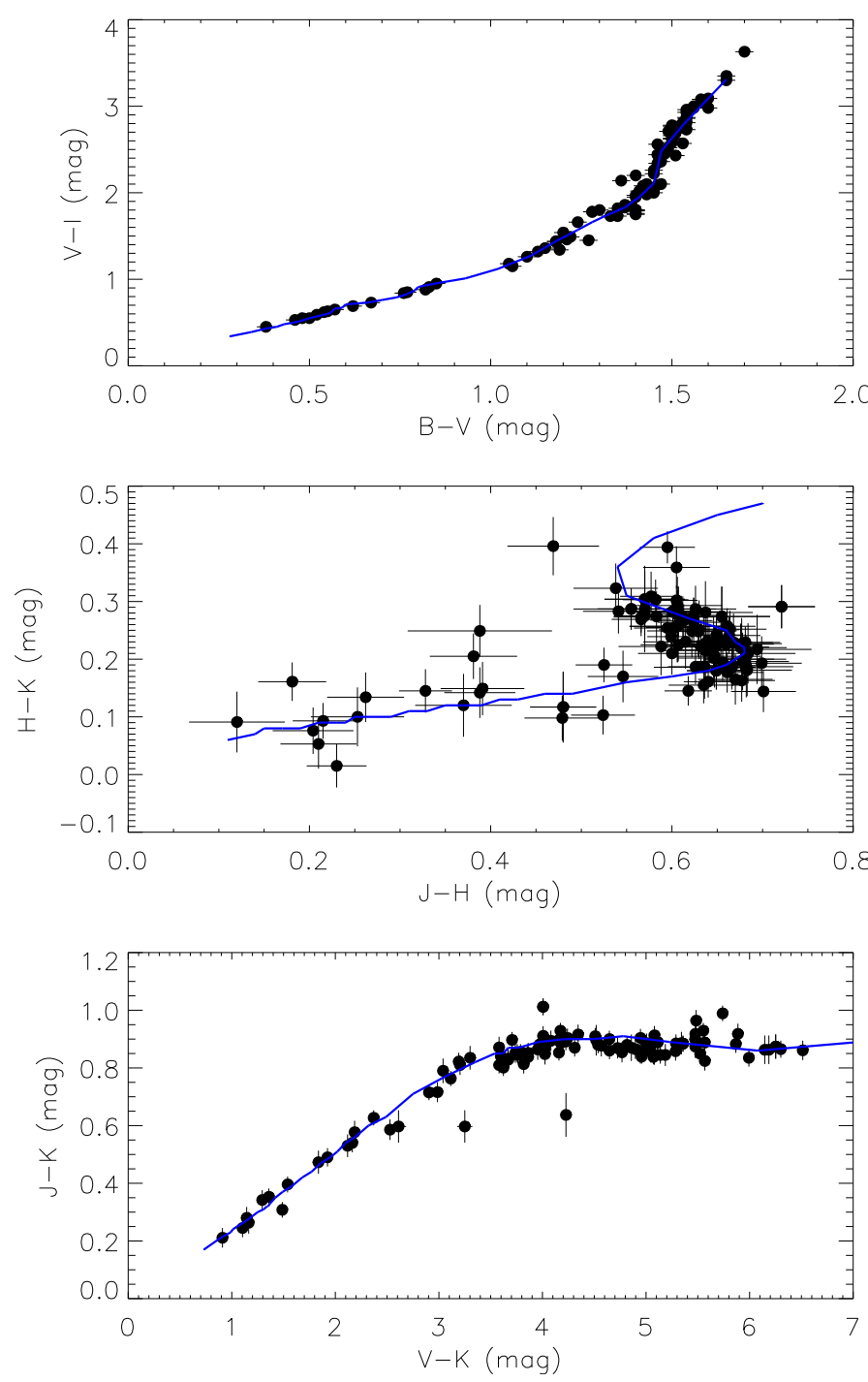

Fig. 1. Color-color plots for the $\beta$ Pictoris members and candidate members with overplotted polynomial fits (blue solid lines) to the corresponding colors taken from Table 6 of Pecaut \& Mamajek (2013).

derived masses, especially for later spectral type stars, significantly depend on the adopted model, with models including effects of magnetic fields giving results more congruent with other age dating methods with respect to non magnetic models (see Messina et al. 2016b, for a detailed discussion). The associated uncertainty on the mass value derives from the uncertainties on distance, apparent magnitude, and effective temperature. In most cases, effective temperatures, which are inferred from spectral types especially for the mid- to late-M stars, have uncertainties not smaller than $\pm 100 \mathrm{~K}$. For this reason, we have investigated which color index is the best stellar mass proxy.

In our sample, $B-V$ and $V-I$ are available for 60 targets; 41 targets have $V-I$ only; 6 targets have $B-V$ only. All these colors are listed in Table A.1 of Paper II and were compiled from different sources in the literature. The remaining ten targets have both $B-V$ and $V-I$ colors unknown. Since the color is a basic parameter in the following analysis, we had to recover the missing values. In the top panel of Fig. 1, we plot the observed $V-I$ versus $B-V$ colors for the program stars. We overplot a polynomial fit to the intrinsic $V-I$ versus $B-V$ colors listed by Pecaut \& Mamajek (2013) for the 5-30 Myr old stars. The agreement is good with an average scatter of 0.05 mag of our colors from the polynomial relation. The agreement mainly arises from the fact that there are a number of $\beta$ Pictoris members in our sample that were used by Pecaut $\&$ Mamajek to infer their tabulated colors for young stars. We used this relation to derive the colors from the measured $V-I$ and $B-V$ colors, respectively, for the mentioned targets missing either $B-V$ or $V-I$, and their associated uncertainty is $0.05 \mathrm{mag}$. For the remaining ten targets with no colors, we inferred them from the spectral type using again the Pecaut \& Mamajek color versus spectral type relations, with an associated uncertainty of $0.07 \mathrm{mag}$. For instance, the distances of our targets have an average value of about $40 \mathrm{pc}$, therefore, the interstellar reddening can be considered negligible and we did not apply any color correction in our analysis.

We note that whereas the $B-V$ color index of our targets spans a range $\Delta \sim 1.35$ of magnitudes, the $V-I$ color index spans a much larger $\Delta \sim 3.3$ mag range, then the latter color index is better suited to represent stars of different masses. On the other hand, in addition to the limit arising from the use of derived $V-I$ colors for about $\sim 70 \%$ of the sample, the two colors come from different works for the majority of stars and they were measured at different epochs. Due to magnetic activity, colors can vary in time up to several hundredths of magnitude ${ }^{1}$ in such a young association. Therefore, the measured colors (and those derived) are not as homogeneous as we would like.

In the middle panel of Fig. 1, we plot the $H-K_{\mathrm{s}}$ versus $J-H$ colors of our targets measured by the 2MASS project (Cutri et al. 2003). We overplot a polynomial fit to the intrinsic $H-K_{\mathrm{s}}$ versus $J-H$ colors listed by Pecaut \& Mamajek (2013) for the 5-30 Myr old stars. We note that a few stars deviate significantly from this relation. They are close binaries with components of different spectral types that were unresolved by 2MASS. We note that the 2MASS color indices of our targets span a range of magnitudes not larger than $\Delta \sim 0.6$, which is too small for our purposes, and, more importantly, the relation is not univocal.

Finally, in the bottom panel of Fig. 1, we plot the $J-K_{\mathrm{s}}$ versus $V-K_{\mathrm{s}}$ colors of our targets measured by 2 MASS, whereas the $V$ mag is the one listed in Table A.1 of Paper II and taken (for 98 out of 117 stars) as the brightest (and presumably unspotted) magnitude in the ASAS (All Sky Automated Survey; Pojmanski 1997) time series or as the brightest magnitude reported in literature (for the remaining 19 stars). Again, we note a few stars deviating significantly from the average trend. We find that the $V-K_{\mathrm{s}}$ color index has a magnitude range of $\Delta \sim 7$ and is the best suited to investigate the color-period distribution. The use of the $V-K_{\mathrm{s}}$ color allows us to deal with an average uncertainty from $\sim 2 \%$ for K0V stars to less than $\sim 0.5 \%$ for late-M stars.

For instance, we note that a comparison with the polynomial fit from Pecaut \& Mamajek (2013) shows that our targets that belong to multiple systems and are unresolved in the 2MASS photometry (separation $\rho \leq 6^{\prime \prime}$ between the components) have $V-K_{\mathrm{s}}$ colors redder on average by $0.03 \mathrm{mag}$ with respect to resolved targets.

\subsection{Rotation period}

The other fundamental stellar property in our investigation is the rotation period. To measure the photometric rotation periods of our targets, we used archive data, we made use of periods from the literature, and carried out our own multi-observatory

\footnotetext{
1 The series of papers on the multiband photometric monitoring of active stars by Cutispoto et al. (e.g., 2003, and references therein), provide an exhaustive example.
} 
observations. A detailed description of the instruments, log of the observations, and information on data reduction and analysis, and the results of the period search are presented in Paper II.

Briefly, in our sample, 52 stars have photometric time series in one or more of the following public archives: ASAS (All Sky Automated Survey; Pojmanski 1997), SuperWASP (Wide Angle Search for Planets; Butters et al. 2010), Integral/OMC (Domingo et al. 2010), HIPPARCos (ESA 1997), NSVS (Northern Sky Variability Survey; Woźniak et al. 2004), MEarth (Berta et al. 2012), and CSS (Catalina Sky Survey; Drake et al. 2009). We have retrieved and analyzed all the available time series for the period search.

Another 20 stars in our sample had no archive data and, thus, they were photometrically monitored by us for the first time. We also observed another 15 stars that, although present in one of the mentioned archives, were either in close binary systems with unresolved components or the archive data did not allow a period determination. We obtained either photometric time series for the resolved components or photometric series suitable for a successful period measurement. For the remaining 30 stars we adopted the rotation periods available in the literature. The results are summarized in Table 2 of Paper II.

To search for the stellar rotation periods of our targets we have followed an approach similar to that used in Messina et al. (2010, 2011). We refer the reader to those papers and to Paper II for a detailed description of the methods.

As a result of our photometric analysis, we obtained the rotation period of 112 out of 117 target stars. Specifically, we measured for the first time the rotation period of 56 stars. For another 27 stars, we confirmed the values reported in the literature with our analysis of new or archived data. For 29 stars we adopted the literature values. For the remaining five stars, our periodogram analysis did not provide the rotation period.

\section{Membership assessment}

For a meaningful investigation of the rotation period distribution and dependences on multiplicity, we first carried out a membership assessment of all 117 stars in our sample by comparing their Galactic velocity $(U V W)^{2}$ relative to the Sun and space $(X Y Z)$ components with respect to the association average values. The proper motions, radial velocities, and distances used to derive $U V W$ and their uncertainties, and $X Y Z$ are listed in Table 2 together with their references. Generally, we found more measurements of RV for each star in the literature and measured a weighted average and its standard deviation for our purposes. Individual RV measurements and relative references are listed in Table 3.

To measure the average values of the Galactic components, we selected an initial sub-sample consisting of stars that were already known as bona fide members of the association and, more precisely, that were investigated in several earlier studies (up to eight for a few; see Paper II) that all agreed to assign the membership to the $\beta$ Pictoris association.

Among these stars, we subsequently selected only single and wide-orbit components of multiple systems to minimize the effect on the derived Galactic components of RV variation arising from orbital motion. In such a way, we were left with 41 stars that represent our "core" sample.

\footnotetext{
$2 U$ positive toward the Galactic centre, $V$ positive in the direction of the Galactic rotation, and $W$ positive in the direction of the Galactic north pole.
}

We use this core sample to compute the average $\bar{U}, \bar{V}$, and $\bar{W}$ velocity components and their standard deviations $\sigma_{U}$, $\sigma_{V}$, and $\sigma_{W}$, and the average $\bar{X}, \bar{Y}$, and $\bar{Z}$ space components and their standard deviations. After computing average values and standard deviations, we found six stars of the core sample that significantly deviated $(>3 \sigma)$ from the other core members in two of three planes $([U, V],[U, W],[V, W])$ : four components of wide binaries (2MASS J02014677+0117161, RBS 269, 2MASS J04435686+3723033, TYC 68721011 1), and two single stars (2MASS J02175601+1225266, 2MASS J164301281754274 with very large uncertainties in their velocity components). These stars were excluded from the core sample and new average values and standard deviations were recomputed as reported in the following:

$$
\begin{aligned}
& \bar{U}\left(\mathrm{~km} \mathrm{~s}^{-1}\right)=-10.27 \pm 1.68 \\
& \bar{V}\left(\mathrm{~km} \mathrm{~s}^{-1}\right)=-15.80 \pm 0.90 \\
& \bar{W}\left(\mathrm{~km} \mathrm{~s}^{-1}\right)=-8.77 \pm 1.20 \\
& \bar{X}(\mathrm{pc})=18 \pm 32 \\
& \bar{Y}(\mathrm{pc})=1 \pm 16 \\
& \bar{Z}(\mathrm{pc})=-20 \pm 7
\end{aligned}
$$

In Fig. 2, we show the 6D kinematic distributions of all 117 stars in our sample. Red bullets represent our core members and the rectangular boxes identify the plane region within $3 \sigma$ from the average values. The values we derived are in agreement within the uncertainties with the values of Torres et al. (2008).

In addition to the kinematics, we used also the Li EW, whenever available (see Paper I for a list of targets with measured $\mathrm{Li}$ EW), as a strong constrain to asses the membership. Those stars in our sample whose $U V W$ and $X Y Z$ differ by less than $3 \sigma$ from the average values of the core sample but whose Li EW significantly deviates $(>3 \sigma)$ from the linear fits to the distribution exhibited by core members (see Fig. 2 in Paper I) are considered non members of the association.

The results of our membership assessment are summarized in Table A.2. As result, in our sample we have 80 bona fide members (flagged with "Y"), of which 35 constituting the core sample (flagged with "Core"), that fully satisfy our criteria for membership, and the above mentioned six bona fide members as reported in the literature, but excluded from our core sample (flagged with "Core_e"). In the present study, we classified as candidate members those stars that have from one to three among space and velocity components deviating more than $3 \sigma$ from the average of the $\beta$ Pictoris association. Whereas, we classified as non members those stars with more than three among space and velocity components deviating more than $3 \sigma$ from the average. Accordingly, in our sample we have 22 candidate members (flagged with "C") and 15 non members (flagged with "NO"). We note that in the following the adjectives "bona fide" and "candidate" only refer to the membership status and not to the single or multiple nature of the targets.

\section{Discussion}

It is unanimously accepted that most if not all low-mass stars form with an accretion protostellar disk that, at early stages, magnetically locks the central star to an about constant angular velocity (e.g., Shu et al. 2000). The disk lifetime has a range of values and stars with a long-lived disk reach the Zero Age Main Sequence rotating more slowly than stars with a short-lived disk. 
S. Messina et al.: Membership and rotation among $\beta$ Pic members
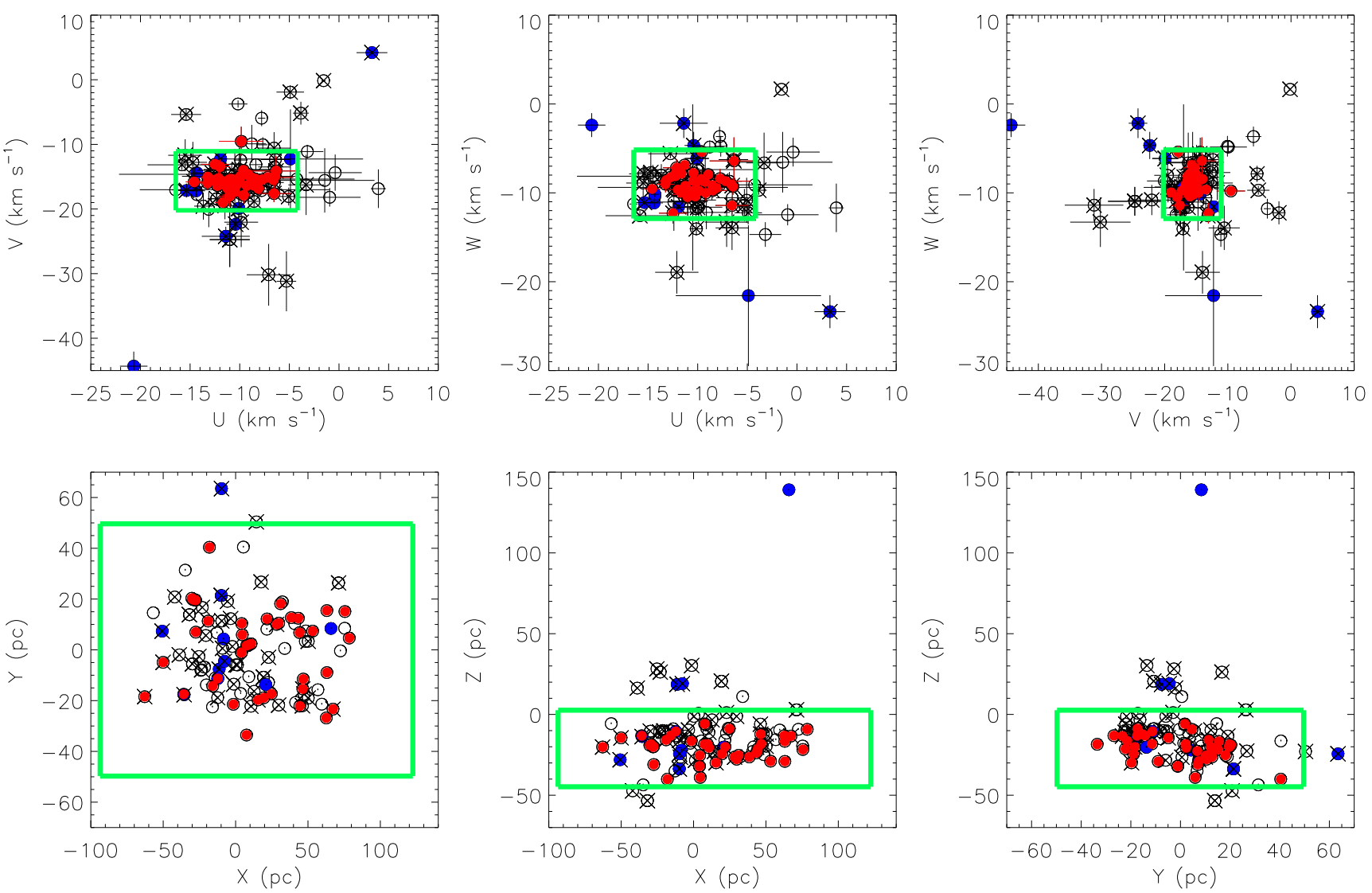

Fig. 2. Distribution of $U V W$ velocity (top panels) and $X Y Z$ space (bottom panels) Galactic components of members and candidate members of the $\beta$ Pictoris association. Red bullets represent the core sample, open bullets the candidate members, blue bullets the rejected members, crossed symbols are for stars with no Li measurement. The green rectangular boxes identify the plane region within $3 \sigma$ from the average values (see text).

Theories predict that the protostellar disk lifetime can be significantly shortened if a binary companion is present, which can truncate the disk, reducing the efficacy of the PMS disk-locking (Meibom et al. 2007; Bouvier et al. 1993; Edwards et al. 1993; Ingleby et al. 2014; Rebull et al. 2004), enhancing the mass accretion (Papaloizou \& Terquem 1995), and finally disrupting the disk (Artymowicz 1992). In this circumstance, the amplitude of the perturbation should be related to the separation between the components. These predictions are confirmed by observational studies, for example by Kraus et al. (2016) and Cieza et al. (2009), who found that stars without IR excess tend to have companions at smaller separation than stars with excess indicating the presence of a disk. Both studies find that the depletion of protoplanetary disks among binary systems with components closer than $40 \mathrm{AU}$ is a factor of two larger than in either single or wide binaries already at age as young as 1-2 Myr. Moreover, if present, disks around close components (<30 AU) of binary systems have disk mass depleted by a factor of 25 with respect to single stars. The impact of a short-lived disks on rotation in binary systems is also documented by, for example, Stauffer et al. (2016) who report that photometric binaries among the Pleiades GKM-type stars tend to rotate faster than their counterpart single stars, with an effect that is more pronounced among equalmass binaries than in single-line spectroscopic binaries; or by Douglas et al. (2016) who report that most, if not all, rapid rotators that deviate from the single-valued relation between mass and rotation already reached by the age of the Hyades, belong to multiple systems.

We are now in the position to extend this investigation of the effect of multiplicity on rotation period distribution to the much younger age of $25 \mathrm{Myr}$, using our sample of $\beta$ Pictoris members and candidate members whose single or multiple nature is well characterized. Moreover, in a sparse system like the $\beta$ Pictoris association, one can assume that stellar encounters have a minor role in altering the stellar angular momentum evolution via disk dissipation or enrichment. Moreover, in the absence of nearby massive stars, disk photo-evaporation by external UV radiation can also be ignored.

We intend to verify that multiplicity really affects the rotational properties and identify the projected separation at which the components of binary or multiple systems of the $\beta$ Pictoris association start to exhibit rotation periods that significantly deviate from the period distribution of single stars.

\subsection{Period distribution of bona fide members}

\subsubsection{Single stars and components of binary or multiple systems}

We start our analysis considering only bona fide members that are single stars and wide components of multiple systems sufficiently distant from each other (projected separation $\rho>$ $500 \mathrm{AU}$ ) to secure that their rotation periods can be considered as they were single stars. In the following, we will show that separations down to $80 \mathrm{AU}$ do not affect significantly the observed rotation periods.

Then, we selected 35 stars: 21 single stars and 14 wide components of multiple systems with $\rho>500 \mathrm{AU}$ (Fig. 3). These stars have rotation periods that exhibit the following mass dependence: the rotation period increases toward lower masses (redder 


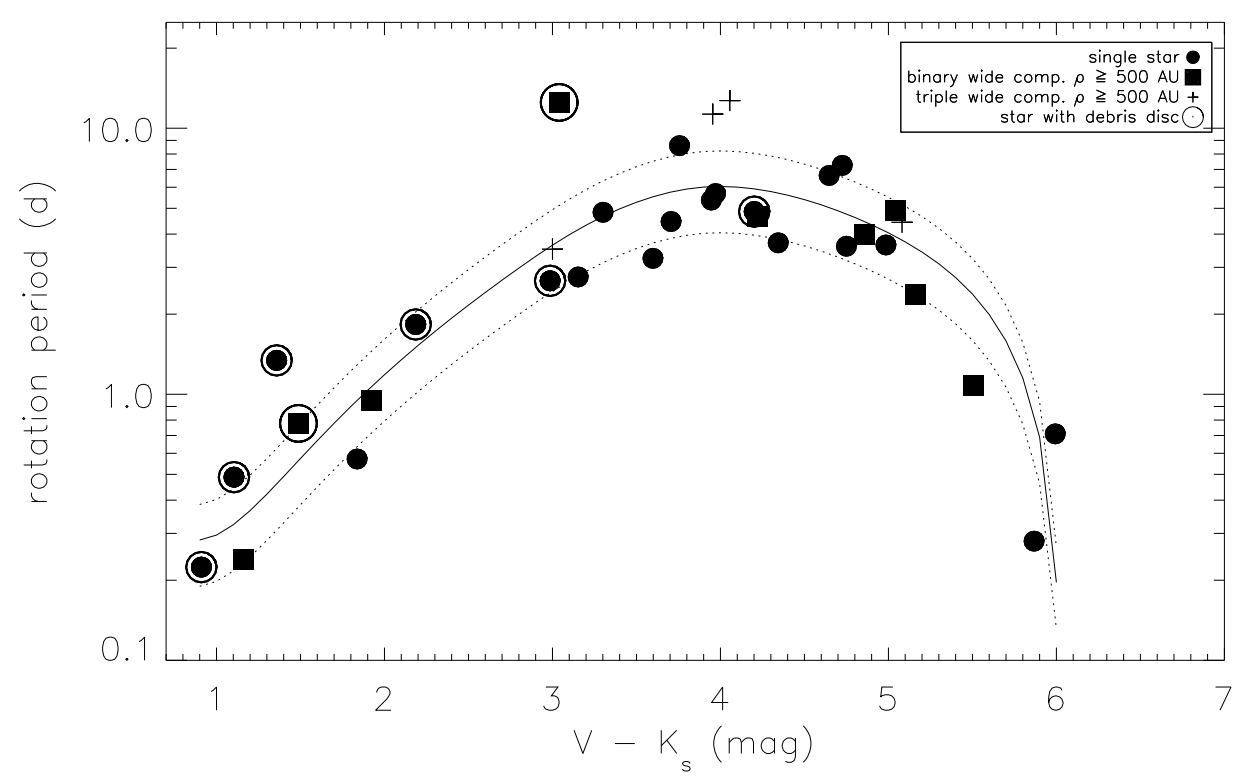

Fig. 3. Distribution versus $V-K_{\mathrm{s}}$ color of the rotation periods of the $\beta$ Pictoris bona fide members that are either single (21 stars) or wide $(\rho>500 \mathrm{AU})$ components of binary or multiple systems (14 stars). The meaning of the symbols is given in the legend. The solid line is a polynomial fit to the rotation periods. Dotted lines represent the $\pm 3 \sigma$ standard deviation of the residuals with respect to the fit.

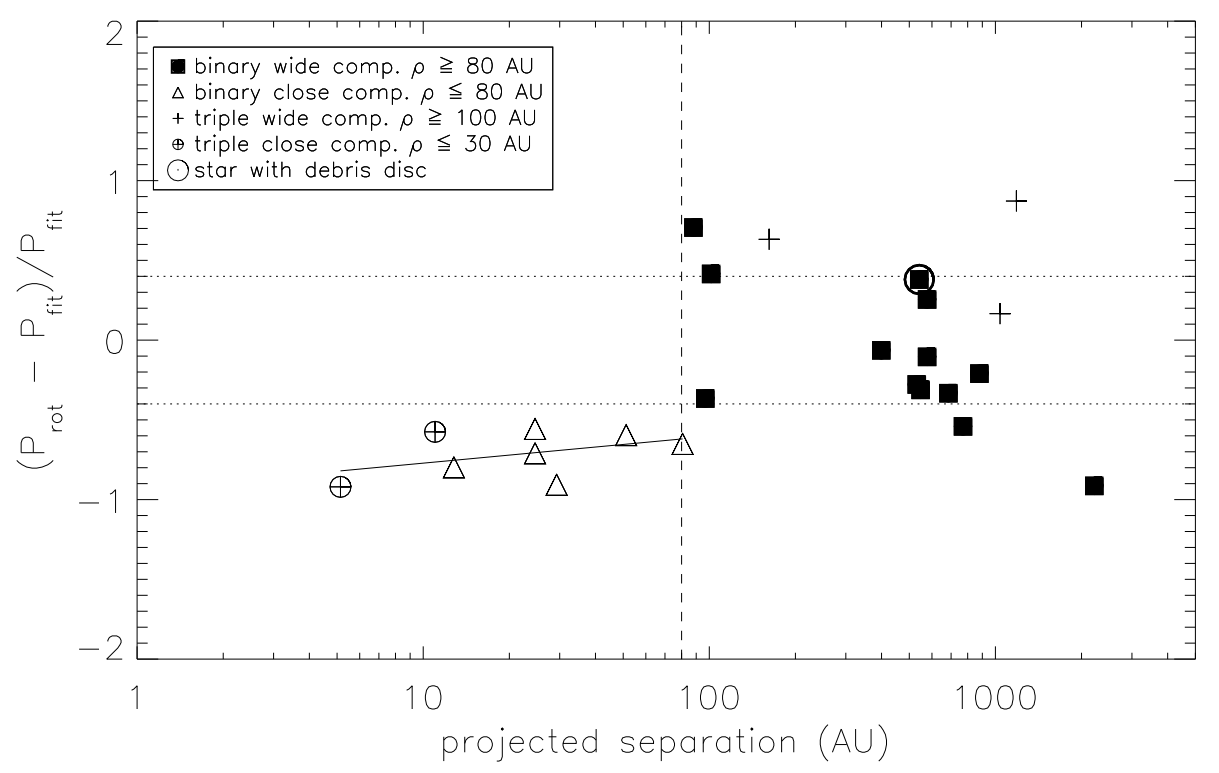

Fig. 4. Relative residuals versus projected separation (AU) of the rotation periods of all bona fide members in binary or multiple systems with respect to the polynomial fit (solid line in Fig. 3). The meaning of the symbols is given in the legend. The solid line is a linear fit to the relative residuals at separation $<80 \mathrm{AU}$. We note that in our sample there are no components of triple systems with separation in the range 30-100 AU.

colors) reaching a maximum at $V-K_{\mathrm{s}} \simeq 4 \mathrm{mag}$, then decreases toward the very-low-mass regime. To measure the mass dependence of the rotation period, we proceeded as follows. We computed the median periods over color bins of 1 mag and computed a polynomial fit to these median values (see Fig. 3) valid in the color range $0.9<V-K_{\mathrm{s}}<6$ mag and whose coefficients are given in Table 1. We find that the relative residuals with respect to the polynomial fit have a normal distribution with a standard deviation $\sigma=0.11$. In the figure we plot the $\pm 3 \sigma$ standard deviation from the $\mathrm{fit}^{3}$. The existence of such a dispersion tells us

3 We excluded from the fit HIP 11437A $\left(V-K_{\mathrm{s}}=3.04\right.$ mag; $P=$ $12.5 \mathrm{~d})$ and HD $160305\left(V-K_{\mathrm{S}}=1.36 \mathrm{mag} ; P=1.341 \mathrm{~d}\right)$ because of their significant $(>20 \sigma)$ departure from the general color-period trend. that the rotation periods of single stars and wide components, in addition to the mass, also depend on other factors such as, for example, differences in the initial rotation periods.

The fit represents empirically the mass dependence of the rotational period of single stars and wide components of binary and multiple systems. The relative residuals $\left(P_{\text {rot }}-P_{\text {fit }}\right) / P_{\text {fit }}$ with respect to this fit can help us to identify which stars deviate significantly and to estimate empirically the minimum separation between the components of a system for which there is no significant departure from this fit. These relative residuals are plotted versus the projected separation (in AU) in Fig. 4. After excluding single stars and very wide components of multiple systems $(\rho>5000 \mathrm{AU})$, and the spectroscopic binaries that will be discussed separately, we find that the components of multiple 


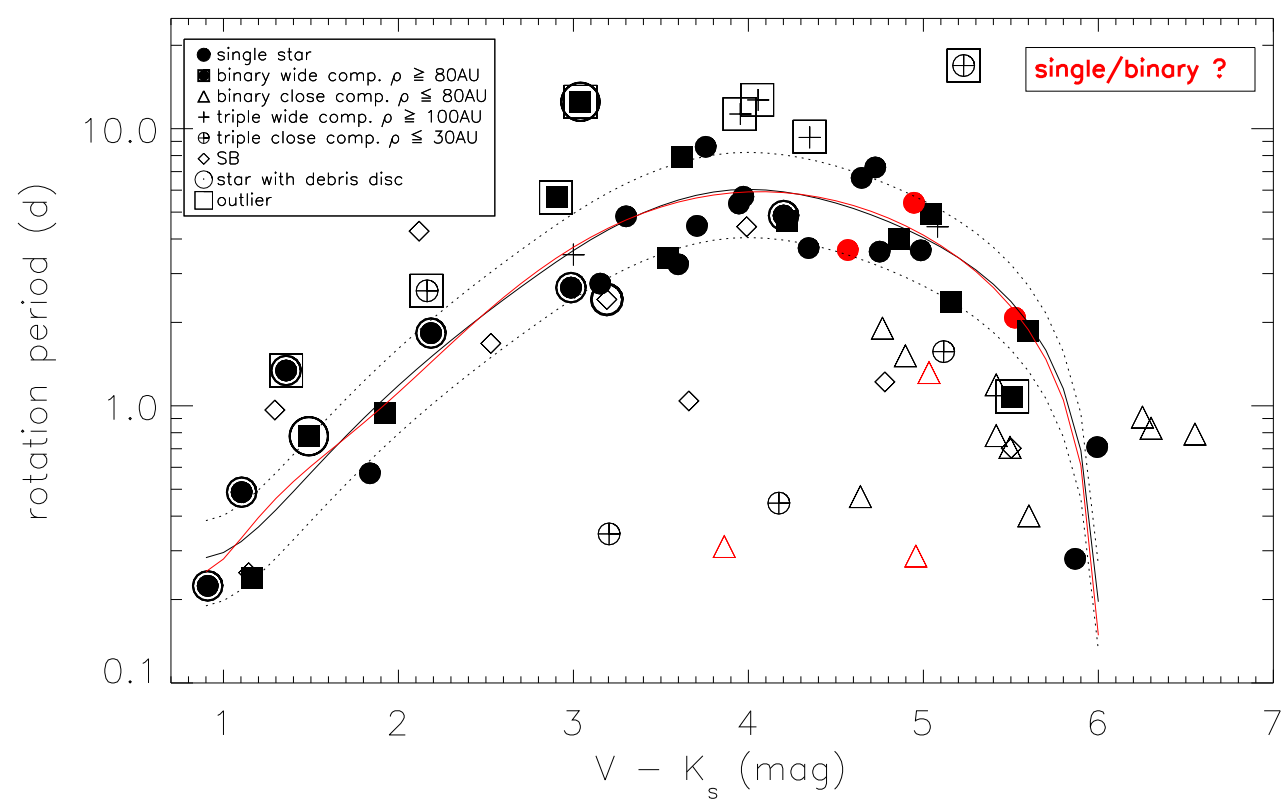

Fig. 5. As in Fig. 3, but with all the bona fide members of the $\beta$ Pictoris association. Open squares indicate the members whose rotation periods significantly deviate either from the general trend exhibited by single stars and components of wide binaries (their residuals from the fit are $>3 \sigma$ ) or from the distribution of visual close binaries. The color red is used for members whose single or binary nature is not known. We note the segregation of all close binaries at rotation periods shorter than the period distribution of single and wide components of multiple systems.

systems with a projected separation $\rho \gtrsim 80 \mathrm{AU}$ are mostly within the $\pm 3 \sigma$ distribution of single stars, therefore they behave as though they were single stars. On the contrary, all components of multiple systems with a projected separation $\rho \lesssim 80$ AU deviate by more than $3 \sigma$. For these residuals, we find a linear Pearson correlation coefficient $r=0.94$ with a significance level $\alpha>99.5 \%$ suggesting that the smaller the separation between the components the faster their rotation period with respect to equal-mass single stars, that is, their rotation periods are significantly affected and shortened. However, the slope of the linear fit to the distribution of residuals for projected separations $\rho \lesssim 80 \mathrm{AU}$

$y=-0.87( \pm 0.24)+0.20( \pm 0.19) \times \log _{10}(\rho)$,

where $y=\left(P_{\text {rot }}-P_{\text {fit }}\right) / P_{\text {fit }}$ has a relatively high uncertainty. Therefore, owing to the paucity of data so far available, we prefer to be more conservative and to state that all components of multiple systems with a projected separation $\rho \lessgtr 80$ AU rotate significantly $(>3 \sigma)$ faster, but a linear dependence of rotation rate on separation is only barely detected.

Among the wide components $(\rho \gtrsim 80 \mathrm{AU})$ we note four stars ${ }^{4}$ (all core members) that deviate more than $3 \sigma$ from the general trend exhibited by the majority of stars. Their departure probably indicates that our scenario, where the separation between the components is the dominant parameter that differentiates the period evolution from that of single stars, is a simplification. There are likely other factors that, in individual cases, may be even more important than the separation.

In Fig. 5, we plot the rotation periods versus $V-K_{\mathrm{s}}$ colors of all bona fide members of the $\beta$ Pictoris association (not only those at $\rho>500 \mathrm{AU}$ as in Fig. 3). In addition to the six mentioned stars (see footnotes 3 and 4), also 2MASS J20013718$3313139\left(V-K_{\mathrm{s}}=4.06 \mathrm{mag} ; P=12.7 \mathrm{~d}\right)$, 2MASS J06131330$2742054\left(V-K_{\mathrm{s}}=5.23 \mathrm{mag} ; P=16.9 \mathrm{~d}\right)$, and TYC 874220651

4 TYC $687801951: V-K_{\mathrm{s}}=2.90 \mathrm{mag}$ and $P=5.70 \mathrm{~d} ; \mathrm{BD}-211074 \mathrm{~A}$ : $V-K_{\mathrm{s}}=4.35 \mathrm{mag}$ and $P=9.3 \mathrm{~d} ;$ TYC $744311021: V-K_{\mathrm{s}}=3.95 \mathrm{mag}$ and $P=11.3 \mathrm{~d}$; TX Psa: $V-K_{\mathrm{s}}=5.57 \mathrm{mag}$ and $P=1.080 \mathrm{~d}$.
Table 1. Coefficients and uncertainties of the polynomial fit to the color-period distribution among bona fide members that are single stars and wide $(\rho>500 \mathrm{AU})$ components of multiple systems in the color range $0.9<V-K_{\mathrm{s}}<6 \mathrm{mag}$.

\begin{tabular}{ccccccccc}
\hline \hline $\mathrm{a} 0$ & $\mathrm{a} 1$ & $\mathrm{a} 2$ & $\mathrm{a} 3$ & $\mathrm{a} 4$ & $\mathrm{a} 5$ & $\mathrm{a} 6$ & $\mathrm{a} 7$ & $\mathrm{a} 8$ \\
\hline 8.38 & -30.90 & 46.59 & -36.37 & 16.16 & -4.14 & 0.60 & -0.046 & 0.00140 \\
\pm 1.75 & \pm 5.09 & \pm 5.99 & \pm 3.73 & \pm 1.36 & \pm 0.30 & \pm 0.04 & \pm 0.002 & \pm 0.00008 \\
\hline
\end{tabular}

$\left(V-K_{\mathrm{s}}=2.16 \mathrm{mag} ; P=2.60 \mathrm{~d}\right)$ deviate significantly from the general color-period trend exhibited by the other members. The existence of these outliers reminds us that in individual cases other factors apart from mass, component's separation, and initial rotation period, may play a significant role in driving the rotational evolution.

\subsubsection{Spectroscopic binaries}

Our stellar sample totals nine spectroscopic binaries (SBs) that are bona fide members (one of which, TYC 740800541 , is an eclipsing binary). Five SBs have known both the components' separation and the orbital periods, which are all shorter than five days and about synchronized with the rotation period of their primary components (the differences amount to a few percents). The star HIP 23418 with a rotation period of $P=1.22 \mathrm{~d}$ against an orbital period $P=11.9 \mathrm{~d}$ represents the only exception. Considering the small $(\rho<0.3 \mathrm{AU})$ component's separation and the almost synchronization between orbital and rotation periods, we infer that tidal dissipation has been effective in these stars, as expected from tidal theory (see, e.g., Zahn 1977; Witte \& Savonije 2002) and as supported by observational studies (see, e.g., Meibom et al. 2007). The tidal dissipation makes their angular momentum evolution different from that of single stars or wide components of binary systems. Considering that the remaining four SBs have same age (being bona fide members), similar total masses, and rotation periods shorter than five 

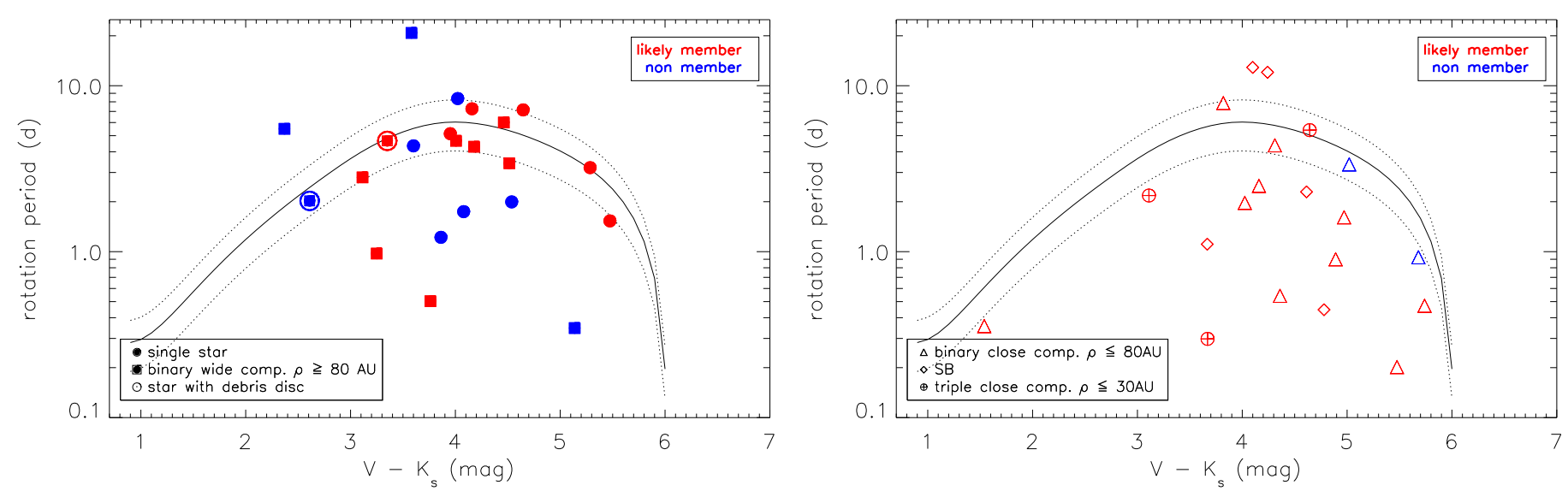

Fig. 6. As in Fig. 5, but for candidate members (red symbols) of the Association and non members (blue symbols). In the left panel we consider single stars and wide components of binary systems; in the right panel we consider the close components of binary systems and spectroscopic binaries.

days, we may suppose that they also are likely significantly affected by tidal dissipation. Because we are focusing our analysis on effects on angular momentum evolution other than tidal dissipation, and the rotation periods of our SBs are not immediately comparable with those of the other stars in our sample, all SBs, but HIP 23418, are excluded from our analysis.

We have only three bona fide members in the very-low-mass regime $\left(V-K_{\mathrm{s}} \geq 6.0 \mathrm{mag}\right)$ that are too red to be compared to the polynomial fit. This part of our color-period diagram is not enough populated to infer any reliable properties.

Finally, in our sample of bona fide members there are six stars (plotted with red symbols) whose single or binary nature is still not determined. We note that 2MASS J08173943$8243298\left(V-K_{\mathrm{s}}=5.03 \mathrm{mag} ; P=1.318 \mathrm{~d}\right), 2$ MASS J17150219. $3333398\left(V-K_{\mathrm{s}}=3.86 \mathrm{mag} ; P=0.3106 \mathrm{~d}\right)$, and $2 \mathrm{MASS}$ $\mathrm{J} 23500639+2659519\left(V-K_{\mathrm{s}}=4.96 \mathrm{mag} ; P=0.287 \mathrm{~d}\right)$ occupy the region of the color-period diagram of close binaries, whereas 2MASS 05015665+0108429 $\left(V-K_{\mathrm{s}}=5.52 \mathrm{mag} ; P=2.08 \mathrm{~d}\right)$, 2MASS J13545390-7121476 $\left(V-K_{\mathrm{s}}=4.57 \mathrm{mag} ; P=3.65 \mathrm{~d}\right)$ and 2MASS J18420694-5554254 $\left(V-K_{\mathrm{s}}=4.95 \mathrm{mag} ; P=\right.$ $5.403 \mathrm{~d}$ ) occupy the region of the color-period diagram of single stars and wide components.

To summarize, the rotation periods of single stars and wide components of multiple systems with separation $\rho \gtrsim 80 \mathrm{AU}$ exhibit a well defined mass dependence at the age of about $25 \mathrm{Myr}$ that can be approximated by a polynomial fit with a dispersion not larger than a factor of two. Only nine bona fide members (see Fig. 5) out of 73 (excluding spectroscopic binaries and very-low mass stars) significantly deviate from the general colorperiod trend exhibited by the other members. The rotation periods of close components of multiple systems with separation $\rho \lesssim 80 \mathrm{AU}$ are all shorter and thus populate the region of the color-period diagram below the distribution of single stars and wide components. When the single or binary nature of the cluster or association members is taken into account, the period distribution even at young ages, such as the presently considered $25 \mathrm{Myr}$, has a spread much smaller than claimed in earlier studies.

\subsection{Period distribution of candidate members}

The sample of bona fide members has allowed us to discover that single stars and wide components of binary or multiple systems have a period distribution different than that of components of close binary and multiple systems. We can take advantage of such a different behavior to infer some suggestion on the candidate members.

\subsubsection{Single stars}

In our sample there are five single candidate members, which are plotted in the left panel of Fig. 6. These candidates have some kinematics component larger than $3 \sigma$ but their rotation periods fit well into the color-period distribution of single bona fide members. We consider these stars as likely members of the association. These stars are 2MASS J16572029-5343316 $\left(V-K_{\mathrm{s}}=4.65 \mathrm{mag} ; P=7.15 \mathrm{~d}\right), 2 \mathrm{MASS} \mathrm{J} 23512227+2344207$ $\left(V-K_{\mathrm{s}}=5.29 \mathrm{mag} ; P=3.208 \mathrm{~d}\right), 2 \mathrm{MASS} \mathrm{J} 16430128-1754274$ $\left(V-K_{\mathrm{s}}=3.95 \mathrm{mag} ; P=5.14 \mathrm{~d}\right.$, which was excluded from the core sample), TYC $585313181\left(V-K_{\mathrm{s}}=4.16 \mathrm{mag} ; P=\right.$ $7.26 \mathrm{~d})$, and 2MASS J05294468-3239141 $\left(V-K_{\mathrm{s}}=5.47 \mathrm{mag}\right.$; $P=1.532 \mathrm{~d})$.

In the left panel of Fig. 6, we also plot the five single stars that are non members. The rotation periods of three of them deviate significantly from the distribution. However, the rotation periods of TYC $91513911\left(V-K_{\mathrm{s}}=3.60 \mathrm{mag} ; P=4.34 \mathrm{~d}\right)$ and 2MASS J20055640-3216591 $\left(V-K_{\mathrm{s}}=4.02 \mathrm{mag} ; P=8.368 \mathrm{~d}\right)$, although non members, fit well into the distribution. This circumstance poses a severe caveat to the use of the rotation period when inferring the age of individual stars. That is, the fact that the rotation period of a single star fits well into the period distribution of the association is a necessary but not sufficient condition to be classified as member.

\subsubsection{Wide components of binary and multiple systems}

In our sample there are seven candidate members that are wide components of multiple systems. These candidates have some kinematics component larger than $3 \sigma$ but the rotation periods of six of them fit well into the color-period distribution of single bona fide members. We consider these stars as likely members of the association. These stars are 2MASS J02014677+0117161 $\left(V-K_{\mathrm{s}}=4.51 \mathrm{mag} ; P=3.41 \mathrm{~d}\right)$, and $\operatorname{RBS} 269\left(V-K_{\mathrm{s}}=\right.$ $4.46 \mathrm{mag} ; P=6.0 \mathrm{~d})$, which were excluded from the core sample, 2MASS J04435686+3723033 $\left(V-K_{\mathrm{s}}=4.18 \mathrm{mag}\right.$; $P=4.288 \mathrm{~d})$, 2MASS J18202275-1011131A $\left(V-K_{\mathrm{s}}=\right.$ $3.35 \mathrm{mag} ; P=4.655 \mathrm{~d})$, 2MASS J18202275-1011131B $(V-$ $\left.K_{\mathrm{s}}=4.01 \mathrm{mag} ; P=5.15 \mathrm{~d}\right)$, and TYC $120804681\left(V-K_{\mathrm{s}}=\right.$ $3.11 \mathrm{mag} ; P=2.803 \mathrm{~d})$. For this star, however, we note that the 


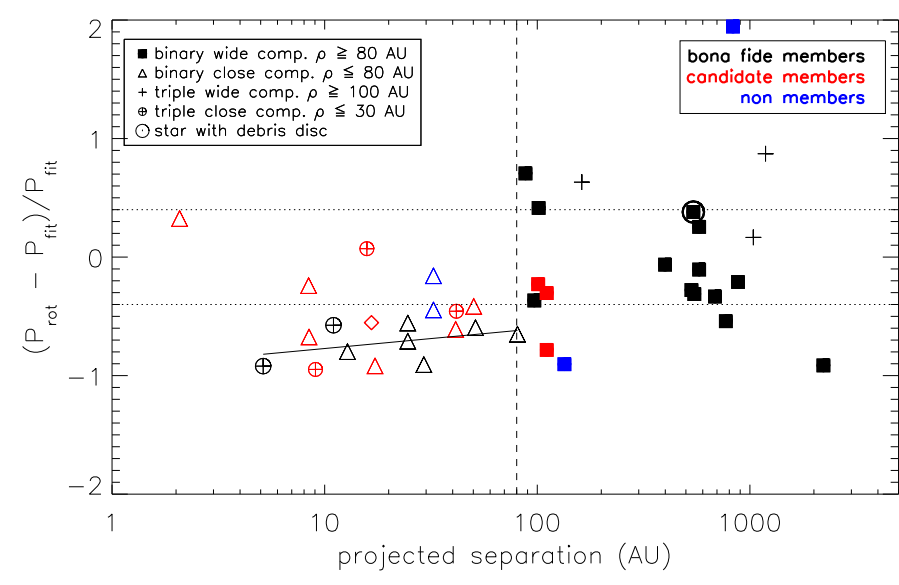

Fig. 7. As in Fig. 4, but with inclusion of candidate members and non members.

rotation period is shorter than that of other members with similar separation ( 100 AU) (see also Fig. 7).

The exception is represented by BD+262161B $\left(V-K_{\mathrm{s}}=\right.$ $3.25 \mathrm{mag} ; P=0.974 \mathrm{~d})$ and TYC $687210111\left(V-K_{\mathrm{s}}=\right.$ 3.76 mag; $P=0.503 \mathrm{~d}$, which was excluded from the core sample) whose rotation periods are in disagreement with the distribution.

In the left panel of Fig. 6, we also plot the five non member wide components: the following stars have accordingly their rotation period in disagreement with the distribution, that is 2MASS J01365516-0647379 $\left(V-K_{\mathrm{s}}=5.14 \mathrm{mag} ; P=\right.$ $0.346 \mathrm{~d})$; HIP105441 $\left(V-K_{\mathrm{s}}=2.37 \mathrm{mag} ; P=5.50 \mathrm{~d}\right)$; and TYC $911412671\left(V-K_{\mathrm{s}}=3.58 \mathrm{mag} ; P=20.8 \mathrm{~d}\right)$. An exception is represented by the debris disk BD $+262161 \mathrm{~A}\left(V-K_{\mathrm{s}}=\right.$ $2.61 \mathrm{mag} ; P=2.022 \mathrm{~d}$ ) whose rotation period is in agreement with the distribution.

\subsubsection{Close components of binary and multiple systems}

In the right panel of Fig. 6, we plot the nine components of close binary systems that are candidate members. All but one of them exhibit rotation periods that are below the distribution of single and wide components, similarly to close components that are bona fide members. However, unlike single stars, this information is not a strong constraint to the membership. For these stars we can state that their rotation period is in agreement with the distribution of the members. However, this is a necessary but not sufficient condition to be considered members. There are other association and clusters with different ages whose period distribution significantly overlap at these short rotation regimes. The only exceptions are HIP 50156 and BD -21 1074B whose rotation periods are too long with respect to the close components members of the $\beta$ Pictoris association. We also plot the three close binary non members, which exhibit rotation periods that tend to be too long and in disagreement with the distribution of the close binary members.

\subsubsection{Spectroscopic binaries}

Our stellar sample totals four spectroscopic binaries that are candidate members. We have information for them on neither orbital period nor on component separation. Also their age is not definite, according to their candidate status. Therefore, we are not in position to infer if their angular momentum has suffered or not significant tidal dissipation. As in Sect. 5.1, we have excluded them from our analysis.

In Fig. 7, we plot the period residuals with respect to the fit versus the projected separation, as in Fig. 4, of all bona fide members, candidate members and non members. As shown, those stars that we classified as likely members follow the distribution exhibited by bona fide members. Our investigation, therefore, supports their candidate membership of the $\beta$ Pictoris association. On the contrary, the following candidate members: HIP $50156\left(\rho=2.08 \mathrm{AU} ;\left(P_{\text {rot }}-P_{\text {fit }}\right) / P_{\text {fit }}=\right.$ $0.325)$, BD $-211074 \mathrm{~B}\left(\rho=15.79 \mathrm{AU} ;\left(P_{\mathrm{rot}}-P_{\mathrm{fit}}\right) / P_{\mathrm{fit}}=\right.$ $0.071), \mathrm{BD}+262161 \mathrm{~B}\left(\rho=110.7 ;\left(P_{\text {rot }}-P_{\text {fit }}\right) / P_{\text {fit }}=-0.78\right)$; TYC $477007971\left(\rho=8.4 \mathrm{AU} ;\left(P_{\text {rot }}-P_{\text {fit }}\right) / P_{\text {fit }}=-0.24\right)$, do not follow the general trend and our study suggests their non membership.

To summarize, the rotation period represents a valuable information when assessing the membership of a star provided that its single or multiple nature and, in the latter case, the separation between the components, are known. The good fitting of the rotation period into the distribution of a proposed association or cluster is a necessary condition for the star to be member, although the rotation period alone does not provide a sufficient condition.

\subsection{Wide components of triple systems}

We note that the wide components of triple systems tend to have rotation periods comparable to but slower than either single stars or components of wide binaries. It seems that in these multiple systems, the initial angular momentum of the protostellar cloud, since it is divided among more components, may have given a fraction of it to the wide component, at least, smaller than that which occurs in the case of binary systems.

The analysis presented in this section shows that, when studying the stellar angular momentum evolution using the rotation period distributions of associations and open clusters, it is fundamental to know the single or binary nature of each member since their rotational properties are significantly different. Mixing single and wide components of multiple systems together with components of close binaries has the consequence to mask the real period segregation between these two different classes of stars, to make the rotation spread to appear larger than it is, and to bias the mean, median, and percentile periods of a given cluster toward smaller values.

\section{Comparison with other open clusters and associations}

A study of the rotation period distribution of the $\beta$ Pictoris members in the context of the angular momentum evolution is out of the scope of this paper. Nonetheless, a comparison with the rotation period distribution of other associations and clusters of different ages is very useful to infer some preliminary and qualitative results, at least, to be further developed elsewhere.

The more recent studies (see, e.g., Messina et al. 2016a) point toward ages of the $\beta$ Pictoris association from 21 to $26 \mathrm{Myr}$. These age estimates are significantly greater than the estimates made by, for example, Zuckerman et al. (2001) and Song et al. (2003), but, for instance, closer to the very first estimate provided by Barrado y Navascués et al. (1999).

The open cluster $h$ Persei (Moraux et al. 2013) with an age of about $13 \mathrm{Myr}$ and the open clusters NGC 2547 (Irwin et al. 2008), IC 2391, IC 2602 (Barnes et al. 1999), and the Argus 
association (Messina et al. 2011), with an age of about $40 \mathrm{Myr}$ have the closest ages to that of $\beta$ Pictoris and have known rotation period distributions. Unfortunately, we face three major limits when comparing their rotation period distributions. Firstly, the single or binary nature of the comparison cluster and association members is not known as accurately as for the $\beta$ Pictoris members. For this reason, we limit the comparison to the upper envelopes of the period distributions, which are likely represented by single stars and wide-orbit binaries. Secondly, we know the rotation periods of only the higher mass members $\left(1<\left(V-K_{\mathrm{s}}\right)_{0}<4 \mathrm{mag}\right)$ of $h$ Persei. Therefore, at the lower mass regime the comparison is possible only at three time steps $(25,40$, and $130 \mathrm{Myr})$. Finally, loose and very sparse associations, as $\beta$ Pictoris, and open clusters may represent two different environments for the dynamical evolution of their members, in the sense that effects of binary encounters on the primordial disk lifetime and, therefore, on the early rotational evolution may be different in magnitude (see Clarke \& Pringle 1993, Heller 1995). Therefore, any results should take into account this major difference.

To compare the distributions, we correct the $V-K_{\mathrm{s}}$ color of the $h$ Per members for interstellar reddening comparing masses and colors taken from Moraux et al. (2013) with the $V-K_{\text {s versus }}$ mass relation for young stars from Pecaut \& Mamajek (2013). As a check, we find that the average color excess $E\left(V-K_{\mathrm{s}}\right)=$ 1.60 mag derived with our approach is in good agreement with the $E\left(V-K_{\mathrm{s}}\right)=1.52 \mathrm{mag}$ inferred from $E(B-V)=0.54 \mathrm{mag}$ (Mayne \& Naylor 2008), assuming $R_{\mathrm{V}}=3.1$. Although we have indication on which members of $h$ Persei are photometric binaries, we do not know the projected separation of their components, therefore we have no possibility to distinguish close from wide orbit binaries, as we did for the $\beta$ Pictoris members. For this reason, we focus on only the single members of $h$ Persei.

We proceed similarly with the NGC 2547 members using the colors and masses provided by Irwin et al. (2008). However, in this case we infer an average color excess $E\left(V-K_{\mathrm{s}}\right)=0.53 \mathrm{mag}$ significantly larger than $E\left(V-K_{\mathrm{s}}\right)=0.20$ mag derived from the $E(B-V)=0.06 \mathrm{mag}$ (Irwin et al. 2008). The reason for this discrepancy is not clear to us. However, irrespectively of the use of the smaller or larger reddening correction, when we overplot the rotation period distribution of the NGC 2547 members on the rotation period distribution of the $\beta$ Pictoris bona fide members, we find qualitatively the same result. For the NGC 2547 members we have no indication on their single or binary nature.

The $V-K_{\mathrm{s}}$ colors of the IC 2391, IC 2602, and Argus members were derived using the $K_{\mathrm{s}}$ magnitudes from 2MASS catalog (Cutri et al. 2003) and $V$ magnitudes from Messina et al. (2011) and Barnes et al. (1999).

The results of the comparison are summarized in the panels of Fig. 8. The comparison period distributions clearly exhibits fast and slow rotators. We use the 90th percentiles computed in 0.5-mag color bins to identify the upper envelope of the rotation period distributions of the comparison cluster and associations. These are represented in the figure ( $h$ Persei in the top left panel, IC 2391+IC 2602+Argus in the bottom left panel, and Pleiades in the bottom right panel) and mark the position of the slowest members.

The slow F-G members of $h$ Persei and of IC 2391+ IC $2602+$ Argus rotate significantly slower than the F-G members of $\beta$ Pictoris. Using the known scenario of PMS angular momentum evolution as guideline, we can infer from Fig. 8 that F-G stars at an age of about 13 Myr ( $h$ Persei members) are still spinning up, owing to radius contraction and angular momentum conservation. They reach a likely maximum rotation rate at an age of about $25 \mathrm{Myr}$ ( $\beta$ Pictoris members), after which they start to slow down, owing to the combined effect of rotation braking by magnetized stellar winds and core-envelope decoupling (see, e.g., Spada et al. 2011), gaining by the age of about 40 Myr the position in the color-period diagram occupied by the IC 2391+IC 2602+Argus slow members. The rotation magnetic braking keeps going with ages as shown, for comparison, by the Pleiades members (bottom panel; Rebull et al. 2016) at an age of about 130 Myr. Such an observational pattern is predicted quite well by models of angular momentum evolution for $1.0 M_{\odot}$ and $0.8 M_{\odot}$ stars. In Fig. 8 we plot the Gallet \& Bouvier (2015) model rotation periods at the sampled ages. In the figure we also indicate the $V-K_{\mathrm{s}}$ colors corresponding to $1.0 M_{\odot}, 0.8 M_{\odot}$, and $0.5 M_{\odot}$ derived from the Baraffe et al. (2008) models used by Gallet \& Bouvier (2015). Some level of disagreement exists for $0.8 M_{\odot}$ stars at $25 \mathrm{Myr}$ and $40 \mathrm{Myr}$, where model periods are shorter than observed.

Among the slow mid-K to early-M stars it is more complicated to retrieve the angular momentum evolution pattern since these stars have distributions that apparently do not differ significantly from each other at the three time steps $25 \mathrm{Myr}$, $40 \mathrm{Myr}$ and $130 \mathrm{Myr}$, giving some hint that the angular momentum evolution of mid-K to early-M stars has been negligible in the 25-130 Myr time interval. The models of angular momentum evolution actually predict a monotonic increase of the rotation rate only from 13 to $40 \mathrm{Myr}$, and about a constant rotation period up to $130 \mathrm{Myr}$ for the $0.5 M_{\odot}$ stars (see, e.g., Gallet \& Bouvier 2015).

Finally, among the mid- to late-M stars we note that the rotation period distributions at the $40 \mathrm{Myr}$ and $130 \mathrm{Myr}$ steps are about indistinguishable and their upper envelope, consisting of the slow rotators, is below the distribution of the $\beta$ Pictoris members. We can interpret this result assuming that mid- to late-M stars undergo the stellar radius contraction until about the age of the Pleiades and, therefore, they are observed to spinning up their rotation period from the age of $\beta$ Pictoris until the Pleiades age, when models of angular momentum evolution predict these stars to reach the maximum rotation rate.

The result that we found for the F and G stars is very important and should be kept in mind when using the rotation period as age indicator. In fact, we found that in the age range from $\sim 13$ Myr to $\sim 40$ Myr the dependence of the rotation period of either single stars or wide components of multiple systems on age is ambiguous. Stars with ages in the 13-25 Myr range (when periods are spinning up) are expected to have again a similar period in the 25-40 Myr range (when periods are slowing down). The uncertainty on the age determination of $\mathrm{F}-\mathrm{G}$ stars will be minimum at $25 \mathrm{Myr}$ and progressively larger as far as we move to younger or older (up to $40 \mathrm{Myr}$ ) ages. On the other hand, such a kind of degeneracy in the age estimate can be successfully removed when the complementary information on the Li EW is available.

\section{Light curve amplitude versus rotation}

The photometric rotational modulation exhibited by all targets arises from the presence of spots unevenly distributed along their stellar longitudes. The peak-to-peak amplitude of this modulation depends on the spot's area, its temperature contrast with respect to the unspotted photosphere, the photometric band, and on a combination of average latitude where spots are located and the inclination of the stellar rotation axis with respect to the observer's line of sight. These last quantities can play in reducing the observed amplitude for a fixed spot area and temperature contrast. 

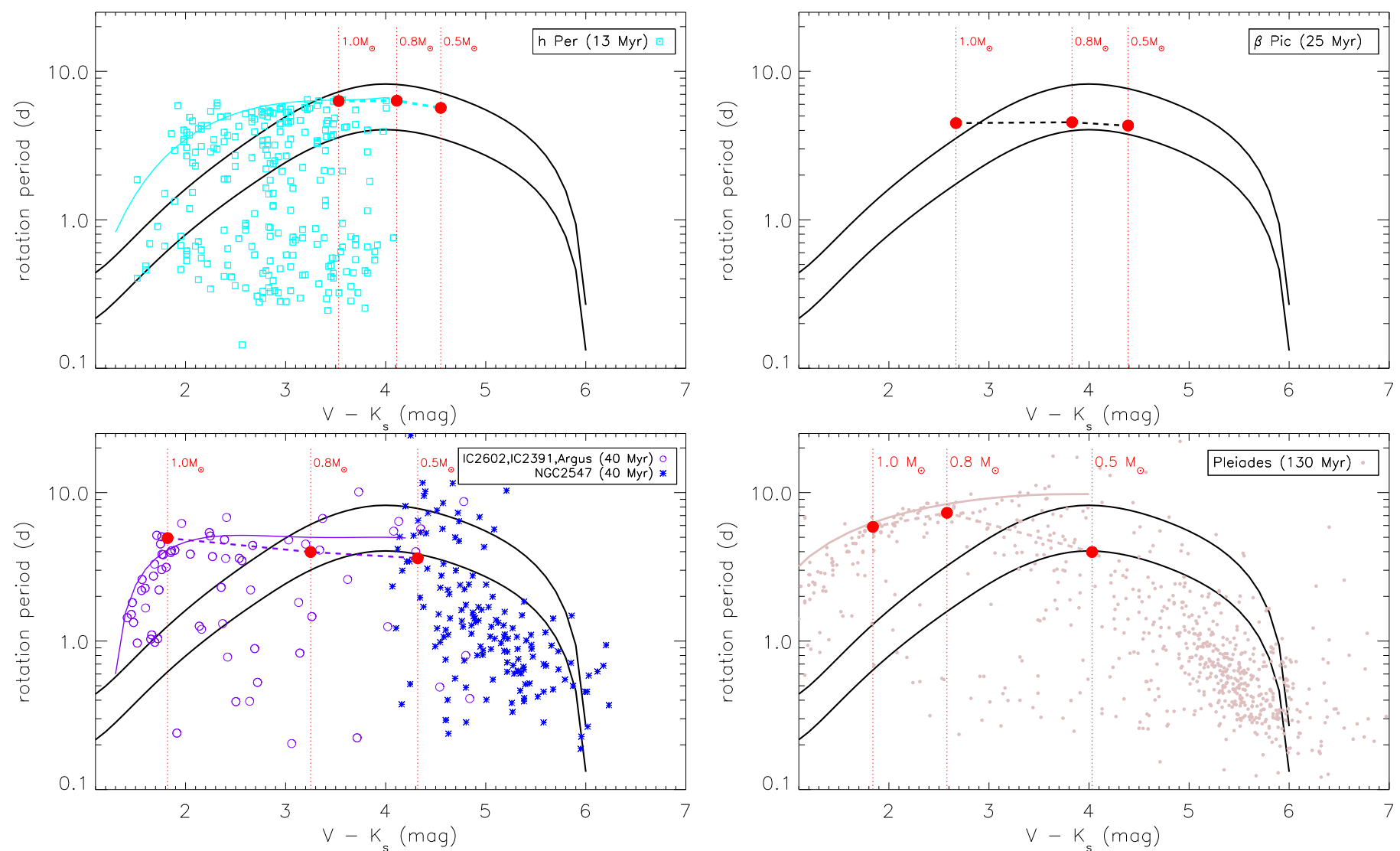

Fig. 8. Rotation period distribution of the $\beta$ Pic members (only the $\pm 3 \sigma$ fits to the distribution are plotted as solid black lines) is compared with the distribution of the $h$ Per single members (light-blue open squares) in the top-left panel; with the distribution of IC 2391+IC 2602+Argus members (violet open circles), and NGC 2547 members (blue asterisks) in the bottom-left panel, and with the distribution of the Pleiades members (brown small bullets) in the bottom-right panel. Thick solid lines are linear fits to the 90th percentile of the distribution of the comparison clusters. Filled bullets connected with dashed lines indicate the model rotation period predicted by the Gallet \& Bouvier (2015) for 1.0, 0.8 and $0.5 M_{\odot}$.

Moreover, the light curve amplitude generally changes versus time on the same star due to active region growth and decay, latitude migration, and presence of spot cycles and/or long-term trends. This is the reason for which stars of similar masses, rotation periods, and ages show a distribution of amplitudes. The amplitude can be then used as an indicator of a lower limit to the level of activity hosted by the star and, when a series of amplitude measurements are available for a given star, the largest value better represents the maximum activity level that the star can exhibit (see, e.g., Messina et al. 2001, 2003).

The correlation between light curve amplitude and rotation period has already been investigated in $\beta$ Pictoris members by Messina et al. $(2010,2011)$ who found no significant correlation. However, the number of available amplitude measurements for each association was not large as in the present case of the $\beta$ Pictoris association. Moreover, in those studies no distinction was made between single or wide components of multiple systems and components of close binaries.

In Fig. 9, the light curve amplitudes of the $\beta$ Pictoris members are plotted versus $\sin i$. Light curve amplitudes, stellar radii, rotation periods, and projected rotational velocities used to derive $\sin i$ are all taken from Paper II. We find that the candidate members that are single or components of wide binaries, and that were found in our previous analysis to have rotation periods that well fit into the period distribution of bona fide members, have a distribution of amplitudes indistinguishable from that of bona fide members. These stars will be also considered in the following analysis. Light curve amplitudes are measured from the amplitude of the sinusoidal fit to the phased light curves. We find with a Kolmogorov test that single stars and wide components of binary or triple systems exhibit the same distribution. This circumstance further confirms that wide components of multiple systems behave as single stars also on the photometric variability point of view.

From the top panel of Fig. 9, we infer that the amplitude is positively correlated with the $\sin i$ with a Spearman rank correlation $\rho=0.53$ and $p$-value $10^{-3}$. This result is expected since equator-on stars $(\sin i=1)$ maximize the amplitude of the rotational modulation of starspots with respect to low-inclination $(\sin i<1)$ stars.

We can use the linear fit to remove the effect of inclination on the amplitude distribution and compute new amplitudes as all stars were equator-on. Then, in the middle panel of Fig. 9, we plot these inclination-corrected amplitudes versus rotation period. We find a Spearman rank correlation $\rho=0.06$ and $p$-value $\sim 0.50$ that allows us to conclude that the amplitude is not correlated to the rotation. This is a very different behavior with respect to older stars, like the AB Doradus and the Pleiades members (see Messina et al. 2001, 2003) whose light curve amplitudes are strongly and negatively correlated to the rotation period. We still note a significant dispersion of the amplitudes around their mean value.

In the bottom panel of Fig. 9, we investigate the dependence of the light curve amplitude on the color, that is, on the stellar mass. Again we find no correlation with a Spearman rank correlation $\rho=0.02$ and $p$-value $\sim 0.30$. Again, the amplitudes show a 

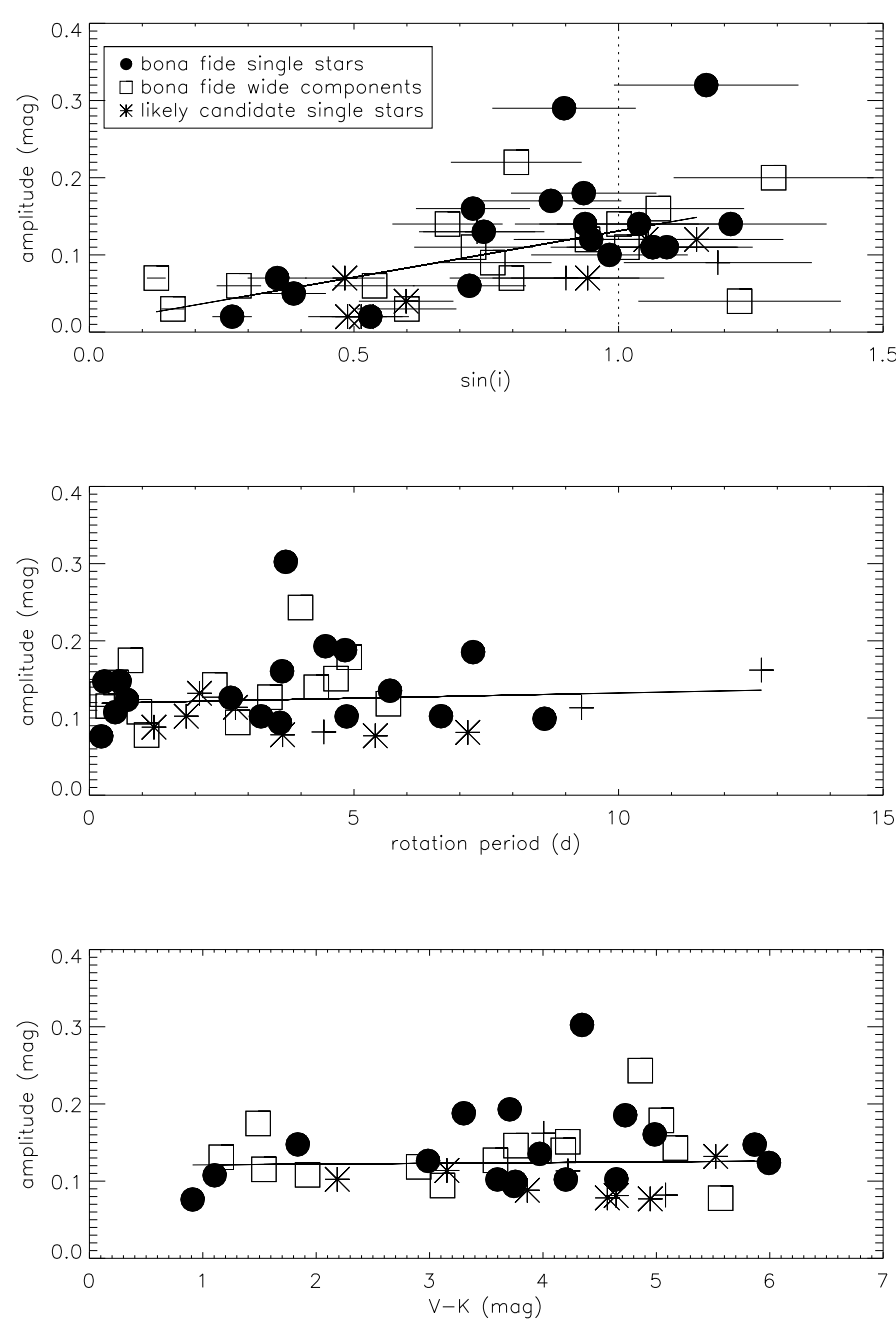

Fig. 9. Top panel: distribution of $V$-band light curve amplitudes versus $\sin i$ for bona fide members that are single stars (bullets), wide components of multiple systems (open squares), and single likely candidate members. Middle panel: same as in the top panel but with amplitudes decorrelated from $\sin i$. Bottom panel: distribution of decorrelated amplitude versus $V-K_{\mathrm{s}}$ color. Solid lines in all panels represent linear fits.

level of dispersion that we attribute to the variable level of activity with time. We note an increase of dispersion, with the highest values around $\mathrm{K}$ and early-M stars.

Similar results are reported by Moraux et al. (2013) for the $h$ Per cluster at the age of 13 Myr. They find the light curve amplitudes to be uncorrelated to the rotation period. Rather, a weak dependence on mass is found, with the lower mass stars having light curve amplitudes slightly larger than higher mass stars. Similarly at the older age of $40 \mathrm{Myr}$, the light curve amplitudes of the NGC 2547 members still appear to be uncorrelated to the rotation period (Irwin et al. 2008). Three single stars TYC 91513911 (with no $v \sin i$ ), TYC 907307621 , and 2MASS J21100535-1919573 all have amplitudes significantly larger than the average $(>0.29 \mathrm{mag})$. These certainly deserve additional study.

\section{Conclusions}

We have assessed the membership of the $\beta$ Pictoris association members using Galactic velocity $(U V W)$ and space $(X Y Z)$ components derived from updated values of proper motions, radial velocities, and distances, complemented with information on $\mathrm{Li}$ content, and rotation period. As result, we have identified 80 bona fide members, 22 candidate members, and 15 non members on a total of 117 stars.

Analyzing the sample of bona fide members, we found that single stars and components of multiple systems with separation larger than about $80 \mathrm{AU}$ have the same distribution of rotation periods vs. the $V-K_{\mathrm{s}}$ color. On the contrary, components of close visual binaries and triples with separation smaller than about $80 \mathrm{AU}$ rotate preferentially faster than their equal-mass single counterparts. This circumstance suggests that when the components are sufficiently close, their primordial disks undergo an enhanced dispersal allowing the stars to start their spin up earlier that single stars.

The characterization of the period distribution made by us and based on bona fide members, has allowed us to infer additional information on candidate members whose single or binary nature is known. As result of this comparison, we find that among our candidate members 17 stars (five single stars, six wide orbit components, and eight close orbit components of multiple system) have rotation periods that further support their membership. On the contrary, three candidate members (one single star and two close components of multiple systems) have rotation periods that favor their non membership.

All but one spectroscopic binaries in our sample have rotation periods that are not immediately comparable with those of either single and wide components or close components of multiple systems since they likely suffered significantly from tidal effects.

A comparison with the rotation period distributions of the younger $h$ Persei open cluster ( $\sim 13 \mathrm{Myr})$ and the older $40-\mathrm{Myr}$ IC 2391, IC 2602, Argus, NGC 2547 and the 130-Myr Pleiades members shows that $F$ and $G$ stars at the age of 13 Myr have not reached yet the zero-age-main-sequence and, therefore, are still contracting their radius spinning up their rotation. They reach a likely maximum rotation rate at the age of about 25 Myr (represented by the $\beta$ Pictoris members). Subsequently, they start a monotonic rotation slowing down which, in our comparison, is readily visible until the Pleiades age. This is the scenario also predicted by models of angular momentum evolution. Unlike the model prediction, the $\mathrm{K}$ and early-M stars in our sample exhibit period distributions that are apparently indistinguishable from each other. That means that in this mass range the single and wide components $\beta$ Pictoris members apparently have rotation periods similar to those of either younger or older stars. However, this mass range in our comparison is not represented as significantly as the $\mathrm{F}$ and $\mathrm{G}$ mass range. Finally, mid- to late-M stars older than $25 \mathrm{Myr}$ all appear to rotate significantly faster than the $\beta$ Pictoris members, giving hint that the rotation spinning up is proceeding in this mass range. Finally, we find that the distribution of light curve amplitudes of single stars is undistinguishable from that of wide components of multiple systems. Moreover, the amplitude is found to increase with $\sin i$, as expected from geometrical considerations. After decorrelating the dependence on $\sin i$, we found no dependence of the amplitude on the rotation period.

Acknowledgements. Research on stellar activity at INAF-Catania Astrophysical Observatory is supported by MIUR (Ministero dell'Istruzione, dell'Università e della Ricerca). This research has made use of the Simbad database, operated at CDS (Strasbourg, France). L.Z. acknowledges support by the Joint Research Fund in Astronomy (U1431114 and U1631236) under cooperative agreement between the National Natural Science Foundation of China and Chinese Academy of Sciences. We are very grateful to the referee whose valuable comments helped us to significantly improve the quality of the paper. 


\section{References}

Alexander, R. 2012, ApJ, 757, L29

Artymowicz, P. 1992, PASP, 104, 769

Baraffe, I., Chabrier, G., \& Barman, T. 2008, A\&A, 482, 315

Barnes, S. A., Sofia, S., Prosser, C. F., \& Stauffer, J. R. 1999, ApJ, 516, 263

Barrado y Navascués, D., Stauffer, J. R., Song, I., \& Caillault, J.-P. 1999, ApJ, 520, L123

Berta, Z. K., Irwin, J., Charbonneau, D., Burke, C. J., \& Falco, E. E. 2012, AJ, 144,145

Beuzit, J.-L., Feldt, M., Dohlen, K., et al. 2008, in Ground-based and Airborne Instrumentation for Astronomy II, Proc. SPIE, 7014, 701418

Biller, B. A., Liu, M. C., Wahhaj, Z., et al. 2013, ApJ, 777, 160

Bouvier, J., Cabrit, S., Fernandez, M., Martin, E. L., \& Matthews, J. M. 1993, A\&A, 272, 176

Bouvier, J., Lanzafame, A. C., Venuti, L., et al. 2016, A\&A, 590, A78

Brandt, T. D., Kuzuhara, M., McElwain, M. W., et al. 2014, ApJ, 786,

Butters, O. W., West, R. G., Anderson, D. R., et al. 2010, A\&A, 520, L10

Chauvin, G., Lagrange, A.-M., Beust, H., et al. 2012, A\&A, 542, A41

Cieza, L. A., Padgett, D. L., Allen, L. E., et al. 2009, ApJ, 696, L84

Clarke, C. J., \& Pringle, J. E. 1993, MNRAS, 261, 190

Cutispoto, G., Messina, S., \& Rodonò, M. 2003, A\&A, 400, 659

Cutri, R. M., Skrutskie, M. F., van Dyk, S., et al. 2003, 2MASS All Sky Catalog of point sources, Vizier Online Data Catalog: II/246

da Silva, L., Torres, C. A. O., de La Reza, R., et al. 2009, A\&A, 508, 833

Desidera, S., Covino, E., Messina, S., et al. 2015, A\&A, 573, A126

Domingo, A., Gutiérrez-Sánchez, R., Rísquez, D., et al. 2010, Astrophys. Space Sci. Proc., 14, 493

Douglas, S. T., Agüeros, M. A., Covey, K. R., et al. 2016, ApJ, 822, 47

Drake, A. J., Djorgovski, S. G., Mahabal, A., et al. 2009, ApJ, 696, 870

Edwards, S., Strom, S. E., Hartigan, P., et al. 1993, AJ, 106, 372

Elliott, P., Bayo, A., Melo, C. H. F., et al. 2014, A\&A, 568, A26

Gallet, F., \& Bouvier, J. 2013, A\&A, 556, A36

Gallet, F., \& Bouvier, J. 2015, A\&A, 577, A98

Heller, C. H. 1995, ApJ, 455, 252

Ingleby, L., Calvet, N., Hernández, J., et al. 2014, ApJ, 790, 47

Irwin, J., Hodgkin, S., Aigrain, S., et al. 2008, MNRAS, 383, 1588

Kiss, L. L., Moór, A., Szalai, T., et al. 2011, MNRAS, 411, 117

Kraus, A. L., Ireland, M. J., Huber, D., Mann, A. W., \& Dupuy, T. J. 2016, AJ, 152,8

Lépine, S., \& Simon, M. 2009, AJ, 137, 3632

Malo, L., Doyon, R., Lafrenière, D., et al. 2013, ApJ, 762, 88

Malo, L., Artigau, É., Doyon, R., et al. 2014a, ApJ, 788, 81

Malo, L., Doyon, R., Feiden, G. A., et al. 2014b, ApJ, 792, 37
Mayne, N. J., \& Naylor, T. 2008, MNRAS, 386, 261

Meibom, S., Mathieu, R. D., \& Stassun, K. G. 2007, ApJ, 665, L155

Messina, S., Rodonò, M., \& Guinan, E. F. 2001, A\&A, 366, 215

Messina, S., Pizzolato, N., Guinan, E. F., \& Rodonò, M. 2003, A\&A, 410, 671

Messina, S., Desidera, S., Turatto, M., Lanzafame, A. C., \& Guinan, E. F. 2010, A\&A, 520, A15

Messina, S., Desidera, S., Lanzafame, A. C., Turatto, M., \& Guinan, E. F. 2011, A\&A, 532, A10

Messina, S., Monard, B., Biazzo, K., Melo, C. H. F., \& Frasca, A. 2014, A\&A, 570, A19

Messina, S., Hentunen, V.-P., \& Zambelli, R. 2015a, IBVS, 6145, 1

Messina, S., Muro Serrano, M., Artemenko, S., et al. 2015b, Ap\&SS, 360, 17

Messina, S., Lanzafame, A. C., Feiden, G. A., et al. 2016a, A\&A, 596, A29 (Paper I)

Messina, S., Naves, R., \& Medhi, B. J. 2016b, New Astron., 48, 5

Messina, S., Millward, M., Buccino, A., et al. 2017, A\&A, 600, A83 (Paper II)

Moraux, E., Artemenko, S., Bouvier, J., et al. 2013, A\&A, 560, A13

Olczak, C., Pfalzner, S., \& Eckart, A. 2010, A\&A, 509, A63

Pallavicini, R., Cutispoto, G., Randich, S., \& Gratton, R. 1993, A\&A, 267, 145

Papaloizou, J. C. B., \& Terquem, C. 1995, MNRAS, 274, 987

Pecaut, M. J., \& Mamajek, E. E. 2013, ApJS, 208, 9

Pojmanski, G. 1997, Acta Astron., 47, 467

Rebull, L. M., Wolff, S. C., \& Strom, S. E. 2004, AJ, 127, 1029

Rebull, L. M., Stauffer, J. R., Bouvier, J., et al. 2016, AJ, 152, 113

Riedel, A. R., Finch, C. T., Henry, T. J., et al. 2014, AJ, 147, 85

Schlieder, J. E., Lépine, S., \& Simon, M. 2010, AJ, 140, 119

Schlieder, J. E., Lépine, S., \& Simon, M. 2012, AJ, 143, 80

Shkolnik, E. L., Anglada-Escudé, G., Liu, M. C., et al. 2012, ApJ, 758, 56

Shu, F. H., Najita, J. R., Shang, H., \& Li, Z.-Y. 2000, Protostars and Planets IV, 789

Song, I., Zuckerman, B., \& Bessell, M. S. 2003, ApJ, 599, 342

Spada, F., Lanzafame, A. C., Lanza, A. F., Messina, S., \& Collier Cameron, A. 2011, MNRAS, 416, 447

Stauffer, J., Rebull, L., Bouvier, J., et al. 2016, AJ, 152, 115

Throop, H. B., \& Bally, J. 2008, AJ, 135, 2380

Torres, C. A. O., Quast, G. R., da Silva, L., et al. 2006, A\&A, 460, 695

Torres, C. A. O., Quast, G. R., Melo, C. H. F., \& Sterzik, M. F. 2008, in Handbook of Star Forming Regions, Vol. II (ASP), ed. Bo Reipurth, 757

Witte, M. G., \& Savonije, G. J. 2002, A\&A, 386, 222

Woźniak, P. R., Vestrand, W. T., Akerlof, C. W., et al. 2004, AJ, 127, 2436

Zahn, J.-P. 1977, A\&A, 57, 383

Zuckerman, B., Song, I., Bessell, M. S., \& Webb, R. A. 2001, ApJ, 562, L87 


\section{Appendix A: Additional tables}

Table A.1. List of $\beta$ Pictoris members analyzed in this study.

\begin{tabular}{|c|c|c|c|c|c|c|c|c|c|}
\hline Target & $\begin{array}{r}\text { RA } \\
(\mathrm{J} 2000)\end{array}$ & $\begin{array}{c}\text { Dec } \\
(\mathrm{J} 2000)\end{array}$ & $\begin{array}{c}V \\
(\mathrm{mag})\end{array}$ & $\begin{array}{l}V-K \\
(\mathrm{mag})\end{array}$ & Sp.T & $\begin{array}{r}P \\
\text { (d) }\end{array}$ & $\begin{array}{l}\Delta P \\
(\mathrm{~d})\end{array}$ & $\begin{array}{r}\Delta V \\
(\mathrm{mag})\end{array}$ & Type \\
\hline HIP 560 & 000650.08 & -230627.20 & 6.15 & 0.91 & F3V & 0.224 & 0.005 & 0.008 & $\mathrm{~S}+\mathrm{D}$ \\
\hline 2MASS J00172353-6645124 & 001723.54 & -664512.50 & 12.35 & 4.65 & $\mathrm{M} 2.5 \mathrm{~V}$ & 6.644 & 0.027 & 0.100 & $\mathrm{~S}$ \\
\hline TYC 118607061 & 002334.66 & 201428.75 & 10.96 & 3.62 & $\mathrm{~K} 7.5 \mathrm{~V}+\mathrm{M} 5$ & 7.9 & 0.1 & 0.070 & $\mathrm{Bw}$ \\
\hline GJ 2006A & 002750.23 & -323306.42 & 12.87 & 4.86 & $\mathrm{M} 3.5 \mathrm{Ve}$ & 3.99 & 0.05 & 0.170 & $\mathrm{Bw}$ \\
\hline GJ 2006B & 002750.35 & -323323.86 & $5 \quad 13.16$ & $5 \quad 5.04$ & $\mathrm{M} 3.5 \mathrm{Ve}$ & 4.91 & 0.05 & 0.120 & $\mathrm{Bw}$ \\
\hline 2MASS J00323480+0729271A & 003234.81 & 072927.10 & 13.40 & 5.02 & M4V & 3.355 & 0.005 & 0.045 & $\mathrm{Bc}$ \\
\hline 2MASS J00323480+0729271B & 003234.81 & 072927.10 & 12.62 & 5.68 & $>\mathrm{M} 5$ & 0.925 & 0.008 & 0.045 & $\mathrm{Bc}$ \\
\hline TYC 585313181 & 010711.94 & -193536.00 & 11.41 & 4.16 & M1V & 7.26 & 0.07 & 0.10 & $\mathrm{~S} ?$ \\
\hline SS J01112542+152 & 011125.42 & 152621.50 & 14.46 & $6 \quad 6.25$ & M5V & 0.911 & 0.001 & 0.01 & $\mathrm{Bc}$ \\
\hline $\mathrm{J} 01112542+1$ & 011125.42 & 152621.50 & 14.46 & $5 \quad 6.55$ & M6V & 0.791 & 0.001 & 0.01 & $\mathrm{Bc}$ \\
\hline 1132817 & 011328.17 & -382102.50 & 11.77 & 4.17( & $(0 \mathrm{~V}+\mathrm{M} 3 \mathrm{~V})+\mathrm{M} 1 \mathrm{~V}$ & 0.446 & - & 0.210 & To \\
\hline 135130 & 013513.93 & -071251.77 & $7 \quad 13.42$ & 5.50 & M4.5V & 0.703 & - & 0.080 & SB2 \\
\hline $2 \mathrm{M}$ & 013655.16 & -064737.92 & 214.00 & 5.14 & $\mathrm{M} 4 \mathrm{~V}+\mathrm{L} 0$ & 0.346 & 0.001 & 0.11 & $\mathrm{Bw}$ \\
\hline TYC 1208046 & 013739.42 & 183532.91 & 9.83 & 3.11 & $\mathrm{~K} 3 \mathrm{~V}+\mathrm{K} 5 \mathrm{~V}$ & 2.803 & 0.010 & 0.07 & $\mathrm{Bw}$ \\
\hline 2MASS J015350 & 015350.77 & -145950.30 & 11.97 & 4.90 & $\mathrm{M} 3 \mathrm{~V}+\mathrm{N}$ & 1.515 & - & 0.110 & $\mathrm{BC}$ \\
\hline $2 \mathrm{M}$ & 02014 & 011716.20 & 12.78 & 4.51 & M & - & - & - & - \\
\hline RBS 269 & 02014 & 011706.00 & 12.72 & 4.46 & M & $98 / 3.30$ & 0.01 & 0.09 & $\mathrm{Bw}$ \\
\hline J02175601+1225266 & 021756.01 & 122526.70 & 13.62 & 4.54 & $\mathrm{M} 3.5 \mathrm{~V}$ & 1.995 & 0.005 & 0.05 & $\mathrm{~S}$ \\
\hline HIP 10679 & 021724.74 & 284430.43 & 37.75 & 1.49 & $\mathrm{G} 2 \mathrm{~V}$ & 0.777 & 0.005 & 0.070 & $B w+D$ \\
\hline HIP 1 & 021725.28 & 284442.16 & 6.95 & 1.16 & & 0.240 & 0.001 & 0.030 & $\mathrm{Bw}$ \\
\hline HIP & 022326.64 & +224406.75 & $5 \quad 11.09$ & 3.74 & M3V & $80 / 3.60$ & 0.02 & 0.06 & $\mathrm{~S}$ \\
\hline HIP 1 & 022729.25 & 305824.60 & 10.12 & 3.04 & $\mathrm{~K} 4 \mathrm{~V}$ & 12.5 & 0.5 & 0.20 & $\mathrm{Bw}+\mathrm{D}$ \\
\hline HIP & 022728.05 & 305840.53 & $3 \quad 12.44$ & 4.22 & & 4.66 & 0.05 & 0.16 & $\mathrm{Bw}$ \\
\hline HIP & 024125.90 & 055918.00 & 10.37 & 3.30 & $\mathrm{~K}$ & 4.83 & 0.03 & 0.180 & $\mathrm{~S}$ \\
\hline 356 & 02.09 & 234235.61 & 17.00 & 5.74 & $\mathrm{M} \varepsilon$ & 0.472 & 0.005 & 0.03 & Bc? \\
\hline 2MASS J034 & 03 & 17.45 & $5 \quad 12.90$ & 4.08 & & & 0.001 & 0.07 & $S$ \\
\hline G & 04 & 28.00 & 10.59 & 4.18 & M1 & 4.89 & 0.01 & 0.05 & $\mathrm{Bc}$ \\
\hline 2MASS J04 & 04 & 372303.30 & 12.98 & 4.18 & M3I & 4.288 & - & - & $\mathrm{Bw}$ \\
\hline & & 700.68 & 310.05 & 3.99 & M0 & 4.430 & 0.030 & 0.150 & SB1 \\
\hline TYC 1281 & 050049.28 & 152700.71 & 10.75 & 3.15 & & 2.76 & 0.01 & & $\mathrm{~S}$ \\
\hline HIP 23309 & 050047.10 & -571525.00 & 10.00 & 3.76 & MoVe & 8.60 & 0.07 & 0.110 & $\mathrm{~S}$ \\
\hline $\mathrm{J} 05015665+0$ & 050156.65 & 010842.91 & 13.20 & 5.52 & & 2.08 & 0.02 & 0.07 & S? \\
\hline HIP 23418A & 050158.80 & 095900.00 & 11.45 & 4.78 & M3V & 1.220 & 0.010 & 0.070 & SB2 \\
\hline HIP 23418B & 050158.80 & 095900.00 & 12.45 & 5.23 & $>\mathrm{M} 3 \mathrm{~V}$ & - & - & - & $\mathrm{Tc}$ \\
\hline $\mathrm{BD}-2$ & 050649.90 & -213509.00 & 10.29 & 4.35 & M & 9.3 & 0.1 & 0.120 & Tw \\
\hline $\mathrm{BD}$ - & 050649.90 & -213509.00 & 11.67 & $7 \quad 4.64$ & $\mathrm{~V}$ & 5.40 & 0.10 & 0.080 & $\mathrm{Tc}$ \\
\hline 2MASS J050 & 050827.30 & -210144.40 & 14.70 & 5.87 & $6 \mathrm{~V}$ & 0.280 & 0.002 & 0.07 & $\mathrm{~S}$ \\
\hline TYC 11 & 052031.83 & +061611.48 & 311.67 & 7311 & K4V & 2.18 & - & 0.09 & To \\
\hline $\mathrm{T}$ & 20 & +061303.57 & $7 \quad 11.58$ & 3300 & $\mathrm{~K}$ & 3.51 & - & 0.08 & $\mathrm{Tw}$ \\
\hline 2MASS J0 & 0 & 30 & 14.32 & 5.60 & $\mathrm{M} 4.5+\mathrm{N}$ & 0.401 & 0.001 & 0.15 & $\mathrm{Bc}$ \\
\hline & 0 & -11 & 6.22 & 1.29 & F7V & 66 & 0.002 & 0.10 & SB2 \\
\hline 2MASS JC & 0 & 20 & 13.79 & 5.47 & $\mathrm{M} \Delta$ & 32 & 0.005 & 0.03 & $\mathrm{~S} ?$ \\
\hline TYC & 204.51 & -030529.38 & 11.32 & 4.31 & $\mathrm{M} 2 \mathrm{~V}+\mathrm{M} 3.5 \mathrm{~V}$ & 4.372 & 0.002 & 0.160 & $\mathrm{Bc}$ \\
\hline 2MASS J05335981-C & 053359.81 & -022132.50 & 12.42 & 4.72 & $\mathrm{M} 2.9 \mathrm{~V}$ & 7.250 & - & 0.170 & $\mathrm{~S}$ \\
\hline 2MASS J06131330-2742054 & 061313.31 & -274205.50 & 12.09 & 5.23 & M3.V: & 16.8 & 1.0 & 0.07 & $\mathrm{Tc}$ \\
\hline HIP 29964 & 061828.20 & -720241.00 & 9.80 & 2.99 & $\mathrm{~K} 4 \mathrm{Ve}$ & 2.670 & 0.010 & 0.120 & $\mathrm{~S}+\mathrm{D}$ \\
\hline 2MASS J07293108+3556003AB & 3072931.09 & 355600.40 & 11.82 & 4.02 & $\mathrm{M} 1+\mathrm{M} 3$ & 1.970 & 0.010 & 0.10 & $\mathrm{Bc}$ \\
\hline 2MASS J08173943-8243298 & 081739.44 & -824329.80 & 11.62 & 5.03 & $\mathrm{M} 3.5 \mathrm{~V}$ & 1.318 & - & 0.050 & $\mathrm{Bc}$ ? \\
\hline 2MASS J08224744-5726530 & 082247.45 & -572653.00 & 13.37 & 5.57 & $\mathrm{M} 4.5+\mathrm{L} 0$ & - & - & - & $\mathrm{Tc}$ \\
\hline 2MASS J09361593+3731456AB & 3093615.91 & 373145.50 & 11.09 & 4.10 & $\mathrm{M} 0.5+\mathrm{M} 0.5$ & 12.9 & 0.3 & 0.030 & SB2 \\
\hline 2MASS J10015995+6651278 & 100200.10 & 665126.00 & 12.38 & 4.16 & M3 & 2.49 & 0.02 & 0.060 & $\mathrm{Bc} ?$ \\
\hline HIP 50156 & 101419.17 & 210429.55 & 510.08 & 33.82 & $10.5 \mathrm{~V}+?$ & 7.860 & - & 0.050 & $\mathrm{Bc}$ \\
\hline TWA 22 & 101726.89 & -535426.50 & 13.99 & 6.30 & M5+M6 & 0.830 & 010 & 0.020 & $\mathrm{Bc}$ \\
\hline $\mathrm{BD}+262161$ & 105938.31 & 252615.50 & 8.45 & 2.61 & $\mathrm{~K} 2$ & 2/0.974 & 0.005 & 0.010 & $\mathrm{Bw}+\mathrm{D}$ \\
\hline $\mathrm{BD}+262161 \mathrm{~B}$ & 105938.31 & 252615.50 & 9.09 & 3.25 & K5 & $0.974 / 2.022$ & 0.005 & 0.010 & $\mathrm{Bw}$ \\
\hline 2MASS J11515681+0731262 & 115156.81 & 073126.25 & 12.38 & 4.61 & $\mathrm{M} 2+\mathrm{M} 2+\mathrm{M} 8$ & 2.291 & - & 0.130 & SB2 \\
\hline 2MASS J13545390-7121476 & 135453.90 & -712147.67 & 712.24 & 4.57 & $\mathrm{M} 2.5 \mathrm{~V}$ & 3.65 & 0.02 & 0.020 & $\mathrm{~S} ?$ \\
\hline HIP & 141421.36 & -152121.75 & 10.27 & 3.67 & $\mathrm{~K} 5.5 \mathrm{~V}+$ & 0.298 & 0.005 & 0.17 & $\mathrm{Tc}$ \\
\hline HIP 69562B & 141421.36 & -152121.75 & 10.27 & 3.67 & - & - & - & - & $\mathrm{Tc}$ \\
\hline
\end{tabular}

Notes. The table lists: name, RA and Dec coordinates, $V$ mag, $V-K_{\mathrm{s}}$ color, spectral type, rotation period and its uncertainty, light curve amplitude, and info on binarity. 
Table A.1. continued.

\begin{tabular}{|c|c|c|c|c|c|c|c|c|c|}
\hline Target & $\begin{array}{r}\mathrm{RA} \\
(\mathrm{J} 2000)\end{array}$ & $\begin{array}{c}\text { Dec } \\
(\mathrm{J} 2000)\end{array}$ & $\begin{array}{c}V \\
(\mathrm{mag})\end{array}$ & $\begin{array}{l}V-K \\
(\mathrm{mag})\end{array}$ & Sp.T & $\begin{array}{l}\mathrm{P} \\
\text { (d) }\end{array}$ & $\begin{array}{l}\Delta P \\
\text { (d) }\end{array}$ & $\begin{array}{r}\Delta V \\
(\mathrm{mag})\end{array}$ & Type \\
\hline TYC 91513911 & 142555.93 & 141210.14 & 10.89 & 3.60 & $\mathrm{~K} 4 \mathrm{~V}$ & 4.340 & - & 0.360 & $S$ \\
\hline HIP 76629 & 153857.50 & -574227.00 & 7.97 & 2.12 & K0V & 4.27 & 0.10 & 0.180 & SB1 \\
\hline 2MASS J16430128-1754274 & 164301.29 & -175427.50 & 12.50 & 3.95 & M0.6 & 5.14 & 0.04 & 0.140 & $\mathrm{~S}$ \\
\hline 2MASS J16572029-5343316 & 165720.30 & -534331.70 & 12.44 & 4.65 & M3V & 7.15 & 0.05 & 0.020 & $\mathrm{~S}$ \\
\hline 2MASS J17150219-3333398 & 171502.20 & -333339.80 & 10.93 & 3.86 & M0V & 0.311 & - & 0.110 & $\mathrm{Bc}$ ? \\
\hline HIP 84586 & 171725.50 & -665704.00 & 7.23 & $2.53 \mathrm{G}$ & G5IV+K5IV & 1.680 & 0.010 & 0.120 & SB2 \\
\hline HD $155555 \mathrm{C}$ & 171731.29 & -665705.49 & 12.71 & 5.08 & $\mathrm{M} 3.5 \mathrm{Ve}$ & 4.43 & 0.01 & 0.070 & $\mathrm{Tw}$ \\
\hline TYC 872822621 & 172955.10 & -541549.00 & 9.55 & 2.19 & K1V & 1.775 & 0.005 & 0.150 & $\mathrm{~S}$ \\
\hline GSC 08350-01924 & 172920.67 & -501453.00 & 13.47 & 4.77 & M3V & 1.906 & 0.005 & 0.05 & $\mathrm{Bc}$ \\
\hline HD 160305 & 174149.03 & -504328.00 & 8.35 & 1.36 & F9V & 1.336 & 0.008 & 0.060 & $\mathrm{~S}+\mathrm{D}$ \\
\hline TYC 874220651 & 174833.70 & -530643.00 & 8.94 & 2.16 & $\mathrm{~K} 0 \mathrm{IV}+$ & $2.60 / 1.62$ & 0.01 & 0.060 & $\mathrm{Tc}$ \\
\hline HIP 88399 & 180303.41 & -513856.43 & 12.50 & $4.23 \mathrm{I}$ & $\mathrm{M} 2 \mathrm{~V}+\mathrm{F} 6 \mathrm{~V}$ & - & - & - & $\mathrm{Bw}$ \\
\hline V4046 Sgr & 181410.50 & -324733.00 & 10.44 & $3.19 \mathrm{l}$ & $\mathrm{K} 5 \mathrm{~V}+\mathrm{K} 7 \mathrm{~V}$ & 2.42 & 0.01 & 0.090 & $\mathrm{SB} 2+\mathrm{D}$ \\
\hline UCAC2 18035440 & 181422.07 & -324610.12 & 12.78 & 4.24 & M1Ve & 12.05 & 0.5 & 0.14 & SB \\
\hline 2MASS J18151564-4927472 & 181515.64 & -492747.20 & 12.86 & 4.78 & M3V & 0.447 & 0.002 & 0.130 & SB1 \\
\hline HIP 89829 & 181952.20 & -291633.00 & 8.89 & 1.84 & G1V & 0.571 & 0.001 & 0.140 & $\mathrm{~S}$ \\
\hline 2MASS J18202275-1011131A & 182022.74 & -101113.62 & 10.63 & 3.35 & $\mathrm{~K} 5 \mathrm{Ve}$ & $4.65 / 5.15$ & - & 0.070 & $\mathrm{Bw}+\mathrm{D}$ \\
\hline 2MASS J18202275-1011131B & 182022.74 & -101113.62 & 10.63 & 4.01 & K7Ve & $5.15 / 4.65$ & - & 0.070 & $\mathrm{Bw}$ \\
\hline 2MASS J18420694-5554254 & 184206.95 & -555425.50 & 13.53 & 4.95 & $\mathrm{M} 3.5 \mathrm{~V}$ & 5.403 & - & 0.070 & $\mathrm{~S}$ ? \\
\hline TYC 907724891 & 184537.02 & -645146.14 & 9.30 & 3.20 & $\mathrm{~K} 8 \mathrm{Ve}$ & 0.345 & 0.005 & 0.160 & $\mathrm{Tc}$ \\
\hline TYC 907307621 & 184652.60 & -621036.00 & 11.80 & 3.95 & M1Ve & 5.37 & 0.04 & 0.320 & $\mathrm{~S}$ \\
\hline HD 173167 & 184806.36 & -621347.02 & 7.28 & 1.14 & F5V & 0.290 & 0.005 & 0.220 & SB1 \\
\hline TYC 740800541 & 185044.50 & -314747.00 & 11.20 & 3.66 & K8Ve & 1.075 & 0.005 & 0.150 & EB \\
\hline HIP 92680 & 185305.90 & -501050.00 & 8.29 & 1.92 & K8Ve & 0.944 & 0.001 & 0.110 & $\mathrm{Bw}$ \\
\hline TYC 687210111 & 185804.20 & -295305.00 & 11.78 & 3.76 & MoVe & 0.503 & 0.004 & 0.060 & $\mathrm{Bw}$ \\
\hline 2MASS J19102820-2319486 & 191028.21 & -231948.60 & 13.20 & 4.99 & M4V & 3.64 & 0.02 & 0.13 & $\mathrm{~S}$ \\
\hline TYC 687801951 & 191144.70 & -260409.00 & 10.27 & 2.90 & K4Ve & 5.70 & 0.05 & 0.090 & $\mathrm{Bw}$ \\
\hline 2MASS J19233820-4606316 & 192338.20 & -460631.60 & 11.87 & 3.60 & MOV & 3.237 & - & 0.110 & S \\
\hline 2MASS J19243494-3442392 & 192434.95 & -344239.30 & 14.28 & 5.50 & M4V & 0.708 & 0.001 & 0.020 & $\mathrm{Bc}$ ? \\
\hline TYC 744311021 & 195604.37 & -320737.71 & 11.80 & 3.95 & $\mathrm{M} 0.0 \mathrm{~V}$ & 11.3 & 0.2 & 0.09 & $\mathrm{Tw}$ \\
\hline 2MASS J19560294-3207186AB & 195602.94 & -320718.70 & 13.30 & 5.12 & M4V & 1.569 & 0.003 & 0.030 & $\mathrm{Tc}$ \\
\hline 2MASS J20013718-3313139 & 200137.18 & -331314.01 & 12.25 & 4.06 & M1V & 12.7 & 0.2 & 0.13 & $\mathrm{Tw}$ \\
\hline 2MASS J20055640-3216591 & 200556.41 & -321659.15 & 11.96 & 4.02 & $\mathrm{M} 2 \mathrm{~V}$ & 8.368 & 0.005 & 0.130 & S \\
\hline HD 191089 & 200905.21 & -261326.52 & 7.18 & 1.10 & F5V & 0.488 & 0.005 & - & $\mathrm{S}+\mathrm{D}$ \\
\hline 2MASS J20100002-2801410AB & 201000.03 & -280141.10 & 13.62 & $4.64 \mathrm{~N}$ & M2.5+M3.5 & 0.470 & 0.005 & 0.040 & $\mathrm{Bc}$ \\
\hline 2MASS J20333759-2556521 & 203337.59 & -255652.20 & 14.87 & 5.99 & $\mathrm{M} 4.5 \mathrm{~V}$ & 0.710 & 0.001 & 0.05 & $\mathrm{~S}$ \\
\hline HIP 102141A & 204151.20 & -322607.00 & 11.09 & 5.42 & M4Ve & 1.191 & 0.005 & 0.040 & $\mathrm{Bc}$ \\
\hline HIP 102141B & 204151.10 & -322610.00 & 11.13 & 5.42 & M4Ve & 0.781 & 0.002 & 0.020 & $\mathrm{Bc}$ \\
\hline 2MASS J20434114-2433534 & 204341.14 & -243353.19 & 12.83 & $4.97 \mathrm{~N}$ & M3.7+M4.1 & 1.610 & 0.010 & 0.03 & $\mathrm{Bc}$ \\
\hline HIP 102409 & 204509.50 & -312027.00 & 8.73 & 4.20 & M1Ve & 4.86 & 0.02 & 0.10 & $\mathrm{~S}+\mathrm{D}$ \\
\hline HIP 103311 & 205547.67 & -170651.04 & 7.35 & 1.54 & F8V & 0.356 & 0.004 & 0.06 & $\mathrm{Bc}$ \\
\hline TYC 634902001 & 205602.70 & -171054.00 & 10.62 & 3.54 & $\mathrm{~K} 6 \mathrm{Ve}+\mathrm{M} 2$ & 3.41 & 0.05 & 0.120 & $\mathrm{Bw}$ \\
\hline 2MASS J21100535-1919573 & 211005.36 & -191957.40 & 11.54 & 4.34 & M2V & 3.71 & 0.02 & 0.29 & $\mathrm{~S}$ \\
\hline 2MASS J21103147-2710578 & 211031.48 & -271057.80 & 15.20 & 5.60 & $\mathrm{M} 4.5 \mathrm{~V}$ & 1.867 & 0.008 & 0.04 & $\mathrm{Bw}$ \\
\hline 2MASS J21103096-2710513 & 211030.96 & -271051.30 & 15.72 & 5.60 & M5V & - & - & - & $\mathrm{Bw}$ \\
\hline HIP 105441 & 212124.49 & -665457.37 & 8.77 & 2.37 & $\mathrm{~K} 2 \mathrm{~V}$ & 5.50 & 0.02 & 0.050 & $\mathrm{Bw}$ \\
\hline TYC 911412671 & 212128.72 & -665506.30 & 10.59 & 3.58 & K7V & 20.5 & 1.0 & 0.015 & $\mathrm{Bw}$ \\
\hline TYC 94869271 & 212527.49 & -813827.68 & 11.70 & 4.36 & M1V & 0.542 & - & 0.190 & $\mathrm{Bc}$ \\
\hline 2MASS J21374019+0137137AB & 213740.19 & 013713.70 & 13.36 & 5.48 & M5V & 0.202 & 0.001 & 0.130 & $\mathrm{Bc}$ \\
\hline 2MASS J21412662+2043107 & 214126.63 & 204310.70 & 13.50 & 4.89 & M3V & 0.899 & 0.001 & 0.03 & $\mathrm{Bc}$ ? \\
\hline TYC 221113091 & 220041.59 & 271513.60 & 11.39 & 3.67 & M0V & 1.109 & 0.001 & 0.080 & $\mathrm{Bc}$ \\
\hline TYC 934004371 & 224248.90 & -714221.00 & 10.60 & 3.71 & K7Ve & 4.46 & 0.03 & 0.16 & S \\
\hline HIP 112312 & 224458.00 & -331502.00 & 12.10 & 5.17 & $\mathrm{M} 4 \mathrm{Ve}$ & 2.37 & 0.01 & 0.110 & $\mathrm{Bw}$ \\
\hline TX Psa & 224500.05 & -331525.80 & 13.36 & 5.57 & $\mathrm{M} 4.5 \mathrm{Ve}$ & 1.080 & 0.005 & 0.030 & $\mathrm{Bw}$ \\
\hline 2MASS J22571130+3639451 & 225711.31 & 363945.14 & 12.50 & 3.86 & M3V & 1.220 & 0.020 & 0.04 & $\mathrm{~S}$ \\
\hline TYC 583206661 & 233230.90 & -121552.00 & 10.54 & 3.97 & MoVe & 5.68 & 0.03 & 0.140 & $\mathrm{~S}$ \\
\hline 2MASS J23500639+2659519 & 235006.39 & 265951.93 & 14.26 & 4.96 & $\mathrm{M} 3.5 \mathrm{~V}$ & 0.287 & 0.005 & 0.05 & $\mathrm{Bc}$ ? \\
\hline 2MASS J23512227+2344207 & 235122.28 & 234420.80 & 14.11 & 5.29 & M4V & 3.208 & 0.004 & 0.060 & $\mathrm{~S}$ \\
\hline
\end{tabular}


Table A.2. Results of membership assessment based on velocity $(U, V, W)$, space $(X, Y, Z)$ components, Li EW, and rotation period $(P)$.

\begin{tabular}{|c|c|c|c|c|c|c|c|c|c|c|}
\hline Target & $U$ & $V$ & $W$ & $X$ & $Y$ & $Z$ & $\mathrm{Li}$ & $P$ & Final & Note \\
\hline HIP 560 & $\mathrm{Y}$ & $\mathrm{Y}$ & $\mathrm{Y}$ & $\mathrm{Y}$ & $\mathrm{Y}$ & $\mathrm{Y}$ & $\mathrm{Y}$ & $\mathrm{Y}$ & $\mathrm{Y}$ & Core \\
\hline 2MASS J00172353-6645124 & $\mathrm{Y}$ & $\mathrm{Y}$ & $\mathrm{Y}$ & $\mathrm{Y}$ & $\mathrm{Y}$ & Y & - & $\mathrm{Y}$ & $\mathrm{Y}$ & Core \\
\hline TYC 118607061 & $\mathrm{Y}$ & $\mathrm{Y}$ & $\mathrm{Y}$ & $\mathrm{Y}$ & $\mathrm{Y}$ & Y & $\mathrm{Y}$ & $\mathrm{Y}$ & $\mathrm{Y}$ & \\
\hline GJ 2006A & $\mathrm{Y}$ & $\mathrm{N}$ & $\mathrm{Y}$ & $\mathrm{Y}$ & $\mathrm{Y}$ & $\mathrm{Y}$ & $\mathrm{Y}$ & Y & Y & Core \\
\hline GJ 2006B & $\mathrm{Y}$ & $\mathrm{Y}$ & $\mathrm{Y}$ & $\mathrm{Y}$ & $\mathrm{Y}$ & $\mathrm{Y}$ & $\mathrm{Y}$ & $\mathrm{Y}$ & Y & Core \\
\hline 2MASS J00323480+072927A & $\mathrm{Y}$ & $\mathrm{Y}$ & $\mathrm{Y}$ & $\mathrm{Y}$ & $\mathrm{Y}$ & $\mathrm{Y}$ & - & $?$ & $\mathrm{~N}$ & \\
\hline 2MASS J00323480+072927B & $\mathrm{Y}$ & $\mathrm{Y}$ & $\mathrm{Y}$ & $\mathrm{Y}$ & $\mathrm{Y}$ & $\mathrm{Y}$ & - & $?$ & $\mathrm{~N}$ & \\
\hline TYC 585313181 & $\mathrm{~N}$ & $\mathrm{~N}$ & $\mathrm{Y}$ & $\mathrm{Y}$ & $\mathrm{Y}$ & $\mathrm{Y}$ & - & Y & Y & $\mathrm{C}$ \\
\hline 2MASS J01112542+1526214A & $\mathrm{Y}$ & $\mathrm{Y}$ & $\mathrm{Y}$ & $\mathrm{Y}$ & $\mathrm{Y}$ & Y & $\mathrm{Y}$ & - & $\mathrm{Y}$ & \\
\hline 2MASS J01112542+1526214B & $\mathrm{Y}$ & $\mathrm{Y}$ & Y & $\mathrm{Y}$ & $\mathrm{Y}$ & Y & - & - & Y & \\
\hline 2MASS J01132817-3821024 & $\mathrm{Y}$ & $\mathrm{Y}$ & $\mathrm{Y}$ & $\mathrm{Y}$ & $\mathrm{Y}$ & $\mathrm{Y}$ & - & Y & Y & \\
\hline 2MASS J01351393-0712517 & $\mathrm{Y}$ & $\mathrm{Y}$ & $\mathrm{Y}$ & $\mathrm{Y}$ & $\mathrm{Y}$ & $\mathrm{Y}$ & $\mathrm{Y}$ & - & $\mathrm{Y}$ & \\
\hline 2MASS J01365516-0647379 & $\mathrm{Y}$ & $\mathrm{Y}$ & $\mathrm{Y}$ & $\mathrm{Y}$ & $\mathrm{Y}$ & $\mathrm{Y}$ & $\mathrm{N}$ & $\mathrm{N}$ & $\mathrm{N}$ & \\
\hline TYC 120804681 & $\mathrm{Y}$ & $\mathrm{Y}$ & $\mathrm{Y}$ & $\mathrm{Y}$ & $\mathrm{Y}$ & $\mathrm{Y}$ & $\mathrm{Y}$ & $\mathrm{N}$ & $\mathrm{Y}$ & $\mathrm{C}$ \\
\hline 2MASS J01535076-1459503 & $\mathrm{Y}$ & $\mathrm{Y}$ & $\mathrm{Y}$ & $\mathrm{Y}$ & $\mathrm{Y}$ & $\mathrm{Y}$ & - & $\mathrm{Y}$ & $\mathrm{Y}$ & \\
\hline 2MASS J02014677+0117161 & $\mathrm{Y}$ & $\mathrm{N}$ & $\mathrm{Y}$ & $\mathrm{Y}$ & $\mathrm{Y}$ & $\mathrm{N}$ & - & $\mathrm{Y}$ & $\mathrm{Y}$ & Core e \\
\hline RBS 269 & $\mathrm{Y}$ & $\mathrm{N}$ & $\mathrm{Y}$ & $\mathrm{Y}$ & $\mathrm{Y}$ & $\mathrm{N}$ & - & $\mathrm{Y}$ & Y & Core_e \\
\hline 2MASS J02175601+1225266 & $\mathrm{Y}$ & $\mathrm{N}$ & $\mathrm{Y}$ & $\mathrm{Y}$ & $\mathrm{Y}$ & $\mathrm{N}$ & - & $\mathrm{N}$ & Y & Core_e \\
\hline HIP 10679 & $\mathrm{Y}$ & $\mathrm{Y}$ & $\mathrm{Y}$ & $\mathrm{Y}$ & $\mathrm{Y}$ & $\mathrm{Y}$ & $\mathrm{Y}$ & Y & Y & Core \\
\hline HIP 10680 & Y & $\mathrm{Y}$ & $\mathrm{Y}$ & $\mathrm{Y}$ & $\mathrm{Y}$ & Y & $\mathrm{Y}$ & Y & Y & Core \\
\hline HIP 11152 & $\mathrm{Y}$ & $\mathrm{Y}$ & $\mathrm{Y}$ & $\mathrm{Y}$ & $\mathrm{Y}$ & $\mathrm{Y}$ & - & Y & Y & Core \\
\hline HIP $11437 \mathrm{~A}$ & $\mathrm{Y}$ & $\mathrm{Y}$ & $\mathrm{Y}$ & $\mathrm{Y}$ & $\mathrm{Y}$ & $\mathrm{Y}$ & $\mathrm{N}$ & $\mathrm{N}$ & Y & Core \\
\hline HIP 11437B & $\mathrm{Y}$ & $\mathrm{Y}$ & $\mathrm{Y}$ & $\mathrm{Y}$ & $\mathrm{Y}$ & $\mathrm{Y}$ & $\mathrm{Y}$ & $\mathrm{Y}$ & Y & Core \\
\hline HIP 12545 & $\mathrm{Y}$ & Y & $\mathrm{Y}$ & $\mathrm{Y}$ & $\mathrm{Y}$ & $\mathrm{Y}$ & $\mathrm{Y}$ & $\mathrm{Y}$ & Y & Core \\
\hline 2MASS J03350208+2342356 & $\mathrm{Y}$ & $\mathrm{N}$ & $\mathrm{Y}$ & $\mathrm{Y}$ & $\mathrm{Y}$ & $\mathrm{Y}$ & - & $\mathrm{Y}$ & Y & $\mathrm{C}$ \\
\hline 2MASS J03461399+1709176 & $\mathrm{N}$ & $\mathrm{N}$ & $\mathrm{N}$ & $\mathrm{Y}$ & $\mathrm{Y}$ & $\mathrm{Y}$ & - & $\mathrm{N}$ & $\mathrm{N}$ & \\
\hline GJ 3305 & $\mathrm{~N}$ & $\mathrm{Y}$ & $\mathrm{Y}$ & $\mathrm{Y}$ & $\mathrm{Y}$ & $\mathrm{Y}$ & $\mathrm{Y}$ & $\mathrm{U}$ & $?$ & $\mathrm{C}$ \\
\hline 2MASS J04435686+3723033 & $\mathrm{Y}$ & $\mathrm{N}$ & $\mathrm{N}$ & $\mathrm{Y}$ & $\mathrm{Y}$ & $\mathrm{Y}$ & $\mathrm{Y}$ & $\mathrm{Y}$ & Y & Core_e \\
\hline HIP 23200 & $\mathrm{Y}$ & $\mathrm{Y}$ & $\mathrm{Y}$ & $\mathrm{Y}$ & $\mathrm{Y}$ & $\mathrm{Y}$ & $\mathrm{Y}$ & - & Y & \\
\hline TYC 128116721 & $\mathrm{Y}$ & $\mathrm{Y}$ & $\mathrm{Y}$ & $\mathrm{Y}$ & $\mathrm{Y}$ & $\mathrm{Y}$ & - & $\mathrm{Y}$ & $\mathrm{Y}$ & \\
\hline HIP 23309 & $\mathrm{Y}$ & $\mathrm{Y}$ & $\mathrm{Y}$ & $\mathrm{Y}$ & $\mathrm{Y}$ & $\mathrm{Y}$ & $\mathrm{Y}$ & $\mathrm{Y}$ & $\mathrm{Y}$ & \\
\hline 2MASS J05015665+0108429 & $\mathrm{Y}$ & Y & $\mathrm{Y}$ & $\mathrm{Y}$ & $\mathrm{Y}$ & $\mathrm{Y}$ & - & Y & Y & \\
\hline HIP $23418 \mathrm{~A}$ & $\mathrm{Y}$ & $\mathrm{Y}$ & $\mathrm{Y}$ & $\mathrm{Y}$ & $\mathrm{Y}$ & $\mathrm{Y}$ & $\mathrm{Y}$ & - & Y & \\
\hline HIP 23418B & $\mathrm{Y}$ & $\mathrm{Y}$ & $\mathrm{Y}$ & $\mathrm{Y}$ & $\mathrm{Y}$ & $\mathrm{Y}$ & - & - & Y & \\
\hline BD $-211074 \mathrm{~A}$ & $\mathrm{Y}$ & $\mathrm{Y}$ & $\mathrm{Y}$ & $\mathrm{Y}$ & $\mathrm{Y}$ & $\mathrm{Y}$ & $\mathrm{Y}$ & $\mathrm{N}$ & $\mathrm{Y}$ & Core \\
\hline BD -21 1074B & $\mathrm{Y}$ & $\mathrm{Y}$ & $\mathrm{N}$ & $\mathrm{Y}$ & $\mathrm{Y}$ & $\mathrm{Y}$ & $\mathrm{Y}$ & $\mathrm{N}$ & $\mathrm{N}$ & $\mathrm{C}$ \\
\hline 2MASS J05082729-2101444 & $\mathrm{Y}$ & $\mathrm{Y}$ & $\mathrm{Y}$ & $\mathrm{Y}$ & $\mathrm{Y}$ & $\mathrm{Y}$ & $\mathrm{Y}$ & Y & $\mathrm{Y}$ & Core \\
\hline TYC 11214861 & $\mathrm{Y}$ & $\mathrm{Y}$ & $\mathrm{Y}$ & $\mathrm{Y}$ & $\mathrm{Y}$ & $\mathrm{Y}$ & - & Y & $\mathrm{Y}$ & \\
\hline TYC 1129171 & $\mathrm{Y}$ & Y & $\mathrm{Y}$ & $\mathrm{Y}$ & $\mathrm{Y}$ & $\mathrm{Y}$ & - & $\mathrm{Y}$ & Y & \\
\hline 2MASS J05241914-1601153 & $\mathrm{Y}$ & $\mathrm{Y}$ & $\mathrm{Y}$ & $\mathrm{Y}$ & $\mathrm{Y}$ & $\mathrm{Y}$ & $\mathrm{Y}$ & Y & Y & \\
\hline HIP 25486 & $\mathrm{Y}$ & $\mathrm{Y}$ & $\mathrm{Y}$ & $\mathrm{Y}$ & $\mathrm{Y}$ & $\mathrm{Y}$ & $\mathrm{Y}$ & - & Y & \\
\hline 2MASS J05294468-3239141 & $\mathrm{N}$ & $\mathrm{N}$ & $\mathrm{N}$ & $\mathrm{Y}$ & $\mathrm{Y}$ & $\mathrm{Y}$ & - & $\mathrm{Y}$ & Y & $\mathrm{C}$ \\
\hline TYC 477007971 & $\mathrm{Y}$ & $\mathrm{Y}$ & $\mathrm{Y}$ & $\mathrm{Y}$ & $\mathrm{Y}$ & $\mathrm{Y}$ & - & $\mathrm{N}$ & $\mathrm{N}$ & $\mathrm{C}$ \\
\hline 2MASS J05335981-0221325 & $\mathrm{Y}$ & Y & $\mathrm{Y}$ & $\mathrm{Y}$ & $\mathrm{Y}$ & $\mathrm{Y}$ & $\mathrm{Y}$ & $\mathrm{Y}$ & $\mathrm{Y}$ & Core \\
\hline 2MASS J06131330-2742054 & $\mathrm{Y}$ & Y & $\mathrm{Y}$ & $\mathrm{Y}$ & $\mathrm{Y}$ & $\mathrm{Y}$ & $\mathrm{Y}$ & $\mathrm{N}$ & Y & \\
\hline HIP 29964 & $\mathrm{Y}$ & $\mathrm{Y}$ & $\mathrm{Y}$ & $\mathrm{Y}$ & $\mathrm{Y}$ & $\mathrm{Y}$ & $\mathrm{Y}$ & Y & Y & Core \\
\hline 2MASS J07293108+3556003AB & $\mathrm{Y}$ & $\mathrm{Y}$ & $\mathrm{Y}$ & $\mathrm{Y}$ & $\mathrm{Y}$ & $\mathrm{N}$ & - & $\mathrm{Y}$ & Y & $\mathrm{C}$ \\
\hline 2MASS J08173943-8243298 & $\mathrm{Y}$ & $\mathrm{Y}$ & $\mathrm{Y}$ & $\mathrm{Y}$ & $\mathrm{Y}$ & Y & - & Y & $\mathrm{Y}$ & \\
\hline 2MASS J08224744-5726530 & $\mathrm{Y}$ & $\mathrm{Y}$ & $\mathrm{Y}$ & $\mathrm{Y}$ & $\mathrm{Y}$ & $\mathrm{Y}$ & - & - & Y & \\
\hline 2MASS J09361593+3731456AB & $\mathrm{Y}$ & $\mathrm{Y}$ & $\mathrm{N}$ & $\mathrm{Y}$ & $\mathrm{Y}$ & $\mathrm{N}$ & - & - & - & $\mathrm{C}$ \\
\hline 2MASS J10015995+6651278 & $\mathrm{Y}$ & $\mathrm{N}$ & $\mathrm{Y}$ & $\mathrm{Y}$ & $\mathrm{Y}$ & $\mathrm{N}$ & - & Y & $\mathrm{Y}$ & $\mathrm{C}$ \\
\hline HIP 50156 & $\mathrm{Y}$ & $\mathrm{N}$ & $\mathrm{N}$ & $\mathrm{Y}$ & $\mathrm{Y}$ & $\mathrm{N}$ & - & $\mathrm{N}$ & $\mathrm{N}$ & $\mathrm{C}$ \\
\hline TWA 22 & $\mathrm{Y}$ & Y & $\mathrm{Y}$ & $\mathrm{Y}$ & $\mathrm{Y}$ & Y & $\mathrm{Y}$ & - & Y & \\
\hline $\mathrm{BD}+262161 \mathrm{~A}$ & $\mathrm{~N}$ & $\mathrm{~N}$ & $\mathrm{~N}$ & $\mathrm{~N}$ & $\mathrm{~N}$ & $\mathrm{~N}$ & - & Y & $\mathrm{N}$ & \\
\hline $\mathrm{BD}+262161 \mathrm{~B}$ & $\mathrm{Y}$ & $\mathrm{Y}$ & $\mathrm{Y}$ & $\mathrm{Y}$ & $\mathrm{Y}$ & $\mathrm{N}$ & - & $\mathrm{N}$ & $\mathrm{N}$ & $\mathrm{C}$ \\
\hline 2MASS J11515681+0731262 & $\mathrm{Y}$ & $\mathrm{Y}$ & $\mathrm{N}$ & $\mathrm{Y}$ & $\mathrm{Y}$ & $\mathrm{N}$ & - & - & & $\mathrm{C}$ \\
\hline 2MASS J13545390-7121476 & $\mathrm{Y}$ & $\mathrm{Y}$ & $\mathrm{Y}$ & $\mathrm{Y}$ & $\mathrm{Y}$ & $\mathrm{Y}$ & - & $\mathrm{Y}$ & Y & \\
\hline HIP 69562A & $\mathrm{Y}$ & $\mathrm{N}$ & $\mathrm{N}$ & $\mathrm{Y}$ & $\mathrm{Y}$ & $\mathrm{N}$ & - & Y & Y & $\mathrm{C}$ \\
\hline
\end{tabular}

Notes. Core: core member; Core_e: core member excluded from fit; C: candidate. 
Table A.2. continued.

\begin{tabular}{|c|c|c|c|c|c|c|c|c|c|c|}
\hline Target & $U$ & $V$ & $W$ & $X$ & $Y$ & $Z$ & $\mathrm{Li}$ & $P$ & Final & Note \\
\hline HIP 69562B & $\mathrm{Y}$ & $\mathrm{N}$ & $\mathrm{Y}$ & $\mathrm{Y}$ & $\mathrm{Y}$ & $\mathrm{N}$ & - & - & $\mathrm{Y}$ & $\mathrm{C}$ \\
\hline TYC 91513911 & $\mathrm{~N}$ & $\mathrm{~N}$ & $\mathrm{~N}$ & $\mathrm{Y}$ & $\mathrm{Y}$ & $\mathrm{N}$ & $\mathrm{Y}$ & $\mathrm{Y}$ & $\mathrm{N}$ & \\
\hline HIP 76629 & Y & $\mathrm{Y}$ & Y & $\mathrm{Y}$ & $\mathrm{Y}$ & $\mathrm{Y}$ & - & - & $\mathrm{Y}$ & \\
\hline 2MASS J16430128-1754274 & $\mathrm{Y}$ & $\mathrm{N}$ & $\mathrm{N}$ & $\mathrm{Y}$ & Y & $\mathrm{N}$ & Y & Y & $\mathrm{Y}$ & Core_e \\
\hline 2MASS J16572029-5343316 & $\mathrm{Y}$ & $\mathrm{Y}$ & $\mathrm{Y}$ & $\mathrm{Y}$ & $\mathrm{Y}$ & $\mathrm{Y}$ & - & $\mathrm{Y}$ & $\mathrm{Y}$ & $\mathrm{C}$ \\
\hline 2MASS J17150219-3333398 & $\mathrm{Y}$ & $\mathrm{Y}$ & $\mathrm{Y}$ & $\mathrm{Y}$ & $\mathrm{Y}$ & $\mathrm{Y}$ & - & $\mathrm{Y}$ & $\mathrm{Y}$ & \\
\hline HIP 84586 & $\mathrm{Y}$ & $\mathrm{Y}$ & $\mathrm{Y}$ & $\mathrm{Y}$ & $\mathrm{Y}$ & $\mathrm{Y}$ & $\mathrm{N}$ & - & $\mathrm{Y}$ & \\
\hline HD $155555 \mathrm{C}$ & $\mathrm{Y}$ & $\mathrm{Y}$ & $\mathrm{Y}$ & $\mathrm{Y}$ & $\mathrm{Y}$ & $\mathrm{Y}$ & $\mathrm{Y}$ & $\mathrm{Y}$ & $\mathrm{Y}$ & Core \\
\hline TYC 872822621 & $\mathrm{Y}$ & $\mathrm{Y}$ & $\mathrm{Y}$ & $\mathrm{Y}$ & $\mathrm{Y}$ & $\mathrm{Y}$ & Y & $\mathrm{Y}$ & $\mathrm{Y}$ & \\
\hline GSC 08350-01924 & $\mathrm{Y}$ & Y & $\mathrm{Y}$ & $\mathrm{Y}$ & $\mathrm{Y}$ & $\mathrm{Y}$ & $\mathrm{Y}$ & $\mathrm{Y}$ & $\mathrm{Y}$ & \\
\hline HD 160305 & $\mathrm{Y}$ & $\mathrm{Y}$ & $\mathrm{Y}$ & $\mathrm{Y}$ & $\mathrm{Y}$ & Y & - & $\mathrm{N}$ & $\mathrm{Y}$ & \\
\hline TYC 874220651 & $\mathrm{Y}$ & $\mathrm{Y}$ & $\mathrm{Y}$ & $\mathrm{Y}$ & $\mathrm{Y}$ & Y & - & $\mathrm{N}$ & $\mathrm{Y}$ & \\
\hline HIP 88399 & $\mathrm{Y}$ & $\mathrm{Y}$ & $\mathrm{Y}$ & $\mathrm{Y}$ & $\mathrm{Y}$ & $\mathrm{Y}$ & - & $\mathrm{Y}$ & Y & Core \\
\hline V4046 Sgr & Y & $\mathrm{Y}$ & Y & $\mathrm{Y}$ & $\mathrm{Y}$ & Y & Y & - & $\mathrm{Y}$ & \\
\hline UCAC2 18035440 & $\mathrm{~N}$ & $\mathrm{~N}$ & $\mathrm{~N}$ & $\mathrm{~N}$ & $\mathrm{~N}$ & $\mathrm{~N}$ & - & - & $\mathrm{N}$ & \\
\hline 2MASS J18151564-4927472 & $\mathrm{N}$ & $\mathrm{Y}$ & $\mathrm{Y}$ & $\mathrm{Y}$ & $\mathrm{Y}$ & $\mathrm{Y}$ & Y & - & - & $\mathrm{C}$ \\
\hline HIP 89829 & $\mathrm{Y}$ & $\mathrm{Y}$ & Y & $\mathrm{Y}$ & $\mathrm{Y}$ & Y & Y & Y & $\mathrm{Y}$ & Core \\
\hline 2MASS J18202275-1011131A & $\mathrm{N}$ & $\mathrm{Y}$ & $\mathrm{Y}$ & $\mathrm{Y}$ & $\mathrm{Y}$ & $\mathrm{N}$ & Y & $\mathrm{Y}$ & $\mathrm{Y}$ & $\mathrm{C}$ \\
\hline 2MASS J18202275-1011131B & $\mathrm{N}$ & $\mathrm{Y}$ & $\mathrm{Y}$ & $\mathrm{Y}$ & $\mathrm{Y}$ & $\mathrm{N}$ & - & $\mathrm{Y}$ & $\mathrm{Y}$ & $\mathrm{C}$ \\
\hline 2MASS J18420694-5554254 & $\mathrm{Y}$ & Y & $\mathrm{Y}$ & $\mathrm{Y}$ & $\mathrm{Y}$ & Y & - & $\mathrm{Y}$ & $\mathrm{Y}$ & \\
\hline TYC 907724891 & $\mathrm{Y}$ & $\mathrm{Y}$ & $\mathrm{Y}$ & $\mathrm{Y}$ & $\mathrm{Y}$ & $\mathrm{Y}$ & $\mathrm{Y}$ & $\mathrm{Y}$ & $\mathrm{Y}$ & \\
\hline TYC 907307621 & $\mathrm{Y}$ & $\mathrm{Y}$ & $\mathrm{Y}$ & $\mathrm{Y}$ & $\mathrm{Y}$ & $\mathrm{Y}$ & $\mathrm{Y}$ & $\mathrm{Y}$ & $\mathrm{Y}$ & Core \\
\hline HD 173167 & Y & $\mathrm{Y}$ & $\mathrm{Y}$ & $\mathrm{Y}$ & $\mathrm{Y}$ & Y & Y & - & $\mathrm{Y}$ & \\
\hline TYC 740800541 & $\mathrm{Y}$ & $\mathrm{Y}$ & Y & $\mathrm{Y}$ & $\mathrm{Y}$ & Y & Y & - & $\mathrm{Y}$ & \\
\hline HIP 92680 & Y & Y & $\mathrm{Y}$ & $\mathrm{Y}$ & $\mathrm{Y}$ & $\mathrm{Y}$ & $\mathrm{Y}$ & $\mathrm{Y}$ & $\mathrm{Y}$ & Core \\
\hline TYC 687210111 & $\mathrm{~N}$ & $\mathrm{Y}$ & $\mathrm{Y}$ & $\mathrm{Y}$ & $\mathrm{Y}$ & $\mathrm{Y}$ & Y & $\mathrm{N}$ & Y & Core_e \\
\hline 2MASS J19102820-2319486 & Y & $\mathrm{Y}$ & $\mathrm{Y}$ & $\mathrm{Y}$ & $\mathrm{Y}$ & Y & Y & $\mathrm{Y}$ & $\mathrm{Y}$ & Core \\
\hline TYC 687801951 & Y & $\mathrm{Y}$ & Y & Y & Y & $\mathrm{Y}$ & $\mathrm{N}$ & $\mathrm{N}$ & $\mathrm{Y}$ & Core \\
\hline 2MASS J19233820-4606316 & Y & $\mathrm{Y}$ & Y & $\mathrm{Y}$ & $\mathrm{Y}$ & $\mathrm{Y}$ & Y & Y & $\mathrm{Y}$ & Core \\
\hline 2MASS J19243494-3442392 & $\mathrm{Y}$ & $\mathrm{Y}$ & $\mathrm{Y}$ & $\mathrm{Y}$ & $\mathrm{Y}$ & $\mathrm{Y}$ & - & $\mathrm{Y}$ & $\mathrm{Y}$ & \\
\hline TYC 744311021 & Y & $\mathrm{Y}$ & $\mathrm{Y}$ & Y & Y & Y & Y & $\mathrm{N}$ & $\mathrm{Y}$ & Core \\
\hline 2MASS J19560294-3207186AB & Y & $\mathrm{Y}$ & Y & Y & Y & $\mathrm{Y}$ & Y & Y & $\mathrm{Y}$ & \\
\hline 2MASS J20013718-3313139 & Y & $\mathrm{Y}$ & Y & Y & Y & $\mathrm{Y}$ & $\mathrm{Y}$ & $\mathrm{N}$ & $\mathrm{Y}$ & Core \\
\hline 2MASS J20055640-3216591 & $\mathrm{N}$ & $\mathrm{N}$ & $\mathrm{N}$ & $\mathrm{N}$ & $\mathrm{N}$ & $\mathrm{N}$ & Y & $\mathrm{Y}$ & $\mathrm{N}$ & \\
\hline HD 191089 & $\mathrm{Y}$ & $\mathrm{Y}$ & $\mathrm{Y}$ & $\mathrm{Y}$ & $\mathrm{Y}$ & $\mathrm{Y}$ & Y & $\mathrm{Y}$ & $\mathrm{Y}$ & Core \\
\hline 2MASS J20100002-2801410AB & $\mathrm{Y}$ & $\mathrm{Y}$ & $\mathrm{Y}$ & $\mathrm{Y}$ & $\mathrm{Y}$ & $\mathrm{Y}$ & $\mathrm{Y}$ & $\mathrm{Y}$ & $\mathrm{Y}$ & \\
\hline 2MASS J20333759-2556521 & $\mathrm{Y}$ & $\mathrm{Y}$ & $\mathrm{Y}$ & $\mathrm{Y}$ & $\mathrm{Y}$ & $\mathrm{Y}$ & $\mathrm{Y}$ & $\mathrm{Y}$ & $\mathrm{Y}$ & Core \\
\hline HIP 102141A & $\mathrm{Y}$ & $\mathrm{Y}$ & $\mathrm{Y}$ & $\mathrm{Y}$ & $\mathrm{Y}$ & $\mathrm{Y}$ & $\mathrm{Y}$ & $\mathrm{Y}$ & $\mathrm{Y}$ & \\
\hline HIP 102141B & $\mathrm{Y}$ & $\mathrm{Y}$ & $\mathrm{Y}$ & Y & $\mathrm{Y}$ & $\mathrm{Y}$ & $\mathrm{Y}$ & $\mathrm{Y}$ & $\mathrm{Y}$ & \\
\hline 2MASS J20434114-2433534 & Y & $\mathrm{N}$ & $\mathrm{N}$ & $\mathrm{Y}$ & $\mathrm{Y}$ & Y & Y & $\mathrm{Y}$ & $\mathrm{Y}$ & $\mathrm{C}$ \\
\hline HIP 102409 & $\mathrm{Y}$ & $\mathrm{Y}$ & $\mathrm{Y}$ & $\mathrm{Y}$ & $\mathrm{Y}$ & $\mathrm{Y}$ & $\mathrm{Y}$ & $\mathrm{Y}$ & Y & Core \\
\hline HIP 103311 & $\mathrm{~N}$ & $\mathrm{Y}$ & $\mathrm{N}$ & $\mathrm{Y}$ & $\mathrm{Y}$ & $\mathrm{Y}$ & $\mathrm{Y}$ & $\mathrm{Y}$ & $\mathrm{Y}$ & $\mathrm{C}$ \\
\hline TYC 634902001 & $\mathrm{Y}$ & Y & Y & Y & Y & $\mathrm{Y}$ & $\mathrm{Y}$ & Y & $\mathrm{Y}$ & Core \\
\hline 2MASS J21100535-1919573 & $\mathrm{Y}$ & $\mathrm{Y}$ & $\mathrm{Y}$ & $\mathrm{Y}$ & $\mathrm{Y}$ & $\mathrm{Y}$ & $\mathrm{Y}$ & $\mathrm{Y}$ & $\mathrm{Y}$ & Core \\
\hline 2MASS J21103147-2710578 & Y & $\mathrm{Y}$ & $\mathrm{Y}$ & Y & Y & Y & Y & $\mathrm{Y}$ & $\mathrm{Y}$ & Core \\
\hline 2MASS J21103096-2710513 & $\mathrm{Y}$ & $\mathrm{Y}$ & $\mathrm{Y}$ & Y & Y & $\mathrm{Y}$ & - & Y & $\mathrm{Y}$ & Core \\
\hline HIP 105441 & $\mathrm{Y}$ & $\mathrm{Y}$ & $\mathrm{Y}$ & $\mathrm{Y}$ & $\mathrm{Y}$ & Y & $\mathrm{N}$ & $\mathrm{N}$ & $\mathrm{N}$ & \\
\hline TYC 911412671 & $\mathrm{Y}$ & $\mathrm{Y}$ & $\mathrm{Y}$ & $\mathrm{Y}$ & $\mathrm{Y}$ & $\mathrm{Y}$ & $\mathrm{N}$ & $\mathrm{N}$ & $\mathrm{N}$ & \\
\hline TYC 94869271 & $\mathrm{~N}$ & $\mathrm{Y}$ & $\mathrm{Y}$ & $\mathrm{Y}$ & $\mathrm{Y}$ & $\mathrm{Y}$ & $\mathrm{Y}$ & $\mathrm{Y}$ & $\mathrm{Y}$ & $\mathrm{C}$ \\
\hline 2MASS J21374019+0137137AB & $\mathrm{Y}$ & $\mathrm{N}$ & $\mathrm{N}$ & $\mathrm{Y}$ & $\mathrm{Y}$ & $\mathrm{Y}$ & - & $\mathrm{Y}$ & $\mathrm{Y}$ & $\mathrm{C}$ \\
\hline 2MASS J21412662+2043107 & $\mathrm{Y}$ & Y & $\mathrm{Y}$ & $\mathrm{Y}$ & $\mathrm{N}$ & $\mathrm{Y}$ & - & $\mathrm{Y}$ & $\mathrm{Y}$ & $\mathrm{C}$ \\
\hline TYC 221113091 & $\mathrm{Y}$ & $\mathrm{N}$ & $\mathrm{Y}$ & $\mathrm{Y}$ & $\mathrm{Y}$ & $\mathrm{Y}$ & $\mathrm{Y}$ & - & - & $\mathrm{C}$ \\
\hline TYC 934004371 & $\mathrm{Y}$ & $\mathrm{Y}$ & $\mathrm{Y}$ & $\mathrm{Y}$ & $\mathrm{Y}$ & $\mathrm{Y}$ & $\mathrm{Y}$ & $\mathrm{Y}$ & $\mathrm{Y}$ & Core \\
\hline HIP 112312 & $\mathrm{Y}$ & $\mathrm{Y}$ & Y & Y & $\mathrm{Y}$ & Y & $\mathrm{Y}$ & Y & $\mathrm{Y}$ & Core \\
\hline TXPsa & $\mathrm{Y}$ & $\mathrm{Y}$ & $\mathrm{Y}$ & $\mathrm{Y}$ & $\mathrm{Y}$ & $\mathrm{Y}$ & $\mathrm{Y}$ & $\mathrm{N}$ & $\mathrm{Y}$ & Core \\
\hline 2MASS J22571130+3639451 & $\mathrm{Y}$ & $\mathrm{N}$ & $\mathrm{N}$ & $\mathrm{Y}$ & $\mathrm{N}$ & $\mathrm{Y}$ & - & $\mathrm{N}$ & $\mathrm{N}$ & \\
\hline TYC 583206661 & Y & $\mathrm{Y}$ & $\mathrm{Y}$ & $\mathrm{Y}$ & $\mathrm{Y}$ & $\mathrm{Y}$ & Y & Y & $\mathrm{Y}$ & Core \\
\hline 2MASS J23500639+2659519 & $\mathrm{Y}$ & Y & $\mathrm{Y}$ & $\mathrm{Y}$ & $\mathrm{Y}$ & $\mathrm{Y}$ & - & $\mathrm{Y}$ & $\mathrm{Y}$ & \\
\hline 2MASS J23512227+2344207 & $\mathrm{Y}$ & $\mathrm{Y}$ & $\mathrm{Y}$ & $\mathrm{Y}$ & $\mathrm{Y}$ & $\mathrm{Y}$ & - & $\mathrm{Y}$ & $\mathrm{Y}$ & $\mathrm{C}$ \\
\hline
\end{tabular}

